\title{
Methodologische Perspektive und methodische Vorgehensweise
}

Wie im nachfolgenden Kapitel im Detail dargestellt, wurden im Forschungsprozess vielfältige Daten erhoben. Alle folgten dem Ziel, die „soziale Veranstaltung“ (Luckmann 1989: 43) des wirtschaftswissenschaftlichen Laborexperiments in seiner natürlichen Eigengesetzlichkeit und seinen unterschiedlichen Dimensionen zu erfassen, um so den Prozess der kommunikativen Konstruktion des wirtschaftswissenschaftlichen Laborexperiments einer gegenstandsadäquaten Analyse zu unterziehen. Während die Ergebnisse der verschiedenen Datenerhebungsmethoden einerseits alle mein ethnographisches Wissen über die Situation bereicherten, war es analytisch lange Zeit problematisch, ein methodisches Verfahren auszumachen, welches fähig war, diese Diversität aufzufangen und zugleich den spezifischen Erklärungsleistungen der unterschiedlichen Daten gerecht zu werden.

Das vierte Kapitel zielt nun darauf ab, den Ablauf des Forschungsprozesses in methodischer und methodologischer Perspektive nachvollziehbar darzulegen. Es gilt einerseits, die genutzten Methoden der Datenerhebung und -analyse darzustellen und zugleich die methodologische Perspektive zu reflektieren und offenzulegen, aus der heraus die Forschung erfolgte. Um eine bessere Übersicht über die unterschiedlichen Aspekte zu erlauben, wurde das Kapitel in drei Abschnitte geteilt. Der erste Teil thematisiert dabei unterschiedliche Geschichtspunkte des Forschungsdesigns (Abschnitt 4.1). Der zweite Teil gibt Auskunft über das Vorgehen der Datenerhebung und die Datengrundlage, auf welcher die empirische Analyse fußt (Abschnitt 4.2). Abschließend wird in einem dritten Teil erläutert, weshalb das Verfahren der wissenssoziologischen Gattungsanalyse für die untersuchte Forschungsfrage sowohl aus methodologischer als auch aus konzeptioneller Sicht geeignet erscheint (Abschnitt 4.3). 


\subsection{Forschungsdesign}

Der Anspruch der Gegenstandsadäquanz als zentrales Gütekriterium für die qualitative Forschung (Steinke 1999, 2017; Flick 1991; Lamnek 1988; Strübing et al. 2018) war dabei sowohl für die Auswahl der passenden konzeptionellen Perspektive und Analysemethode als auch für die Suche nach geeigneten Verfahren der Datenerhebung und -auswertung forschungsleitend. Im Sinne der Gegenstandsadäquanz besteht das zentrale Anliegen darin, eine passende Methode für das Forschungsdesign, die Erhebung und die Datenanalyse zu finden, die den Eigenheiten des zu untersuchenden Forschungsgegenstands entspricht. In meinem eigenen Forschungsprozess stellte dieser Anspruch der Gegenstandsadäquanz der Methode einen zentralen Orientierungspunkt in der Auseinandersetzung mit dem Feld der experimentellen Wirtschaftswissenschaft und dem spezifischen Phänomen des Laborexperiments dar. Die Anwendung unterschiedlicher Methoden der Datenerhebung waren dabei Schritte einer methodologischen Suchbewegung mit dem Ziel, die jeweils passende Methode zu finden, um sich meinem Forschungsgegenstand so weit wie möglich anzunähern und den unterschiedlichen Aspekten des Phänomens in adäquater Weise Rechnung zu tragen.

Im Zuge dieses Kapitels werden die einzelnen Schritte der Datenerhebung dabei überblicksartig in ihrer zeitlichen Abfolge vorgestellt. Auf diese Weise wird ersichtlich, wie sich die schrittweise Annäherung an den Forschungsgegenstand vollzog und welchen Einfluss der Projektzusammenhang, in welchen ich eingebunden war, auf die Datenerhebung ausübte. Zugleich wird die methodologische Perspektive reflektiert, aus der heraus diese Forschungsarbeit umgesetzt wurde. Im Sinne der Grounded Theory (Strauss \& Corbin 1996; Strübing 2014) kann der Weg der Auseinandersetzung mit dem Forschungsgegenstand dabei als ein iterativ-zyklischer Forschungsprozess beschrieben werden.

Nachfolgend wird ausgeführt, dass meine Forschungsarbeit im Sinne einer allgemeinen Einordnung als eine ethnographische Untersuchung betrachtet werden kann. Im Zuge der Untersuchung kristallisierte sich jedoch eine Zuspitzung auf die Herstellung der typischen Situation des ökonomischen Laborexperiments und die konstitutiven kommunikativen Prozesse heraus. Aus diesem Grund scheint die „fokussierte Ethnographie“ (Knoblauch 2001) das Forschungsvorgehen in späteren Phasen der Forschung passender zu charakterisieren. Im Rahmen dieses Methodenkapitels wird dabei auch ersichtlich, warum die Gattungsanalyse als wissenssoziologische Methode - auf der Basis der Auseinandersetzung mit dem empirischen Phänomen - für die Analyse ausgewählt wurde. Der entscheidende Vorteil besteht dabei in der Chance, die erhobenen Daten in einer Art und Weise zu triangulieren, die den Eigenwert der unterschiedlichen Datensorten 
gewinnbringend zur Klärung der verschiedenen Facetten und Ebenen des Phänomens nutzt. Das ,qualitative Experiment“ (Abschnitt 4.1.3), welches im Rahmen dieses ersten Teils des Methodenkapitels eingeführt wird, ist dabei einerseits als Ergänzung zu den anderen methodischen Erhebungsverfahren zu verstehen, und andererseits als das Resultat der Auseinandersetzung einer qualitativen Forscherin mit dem Feld der quantitativen Laborexperimente. Im Abschluss des Analysekapitels wird anhand einer Serie eigener Experimente eine Anwendung des qualitativen Experiments für die Forschung im Experimentallabor illustriert (Abschnitt 5.5).

\subsubsection{Reflexive Betrachtung der eigenen Forschungsposition}

Sowohl die Daten, die dieser Arbeit zugrunde liegen, als auch die Fragestellung der Arbeit selbst ist deutlich durch das Projekt beeinflusst, in welchem ich in den letzten Jahren als wissenschaftliche Mitarbeiterin tätig war. Dieser Abschnitt dient einerseits dazu, meine Forschungsposition aus einer reflexiven Perspektive einzuordnen und diesen Aspekt als Einflussfaktor im Forschungsprozess klar $\mathrm{zu}$ artikulieren. Zugleich wird so eine bessere Einordnung der nachfolgenden Ausführungen über die unterschiedlichen Wege der Datenerhebung ermöglicht.

Als wissenschaftliche Mitarbeiterin arbeitete ich in einem interdisziplinären Projekt, welches am Wissenschaftszentrum Berlin für Sozialforschung (WZB) angesiedelt war und den Titel „Kulturell bedingte Framing-Effekte in der experimentellen Spieltheorie“ trug. Sowohl organisatorisch als auch inhaltlich zielte das Projekt darauf ab, zwei Abteilungen des WZB, „Verhalten auf Märkten“ und „Kulturelle Quellen von Neuheit“, zum wissenschaftlichen Austausch anzuregen. Aus diesem Grund wurde es als „Brückenprojekt“ bezeichnet. Die DirektorInnen der Abteilungen, Prof. Dorothea Kübler und Prof. Michael Hutter, nahmen dabei zugleich die Rolle der Projektleitung ein. Die Projektgruppe wurde jeweils durch eine wissenschaftliche MitarbeiterIn und die DirektorIn gebildet. Während meine Seite des Projekts durch eine qualitative soziologische Forschungsperspektive charakterisiert wurde, handelte es sich bei unseren ForschungspartnerInnen um Forschende aus dem Bereich der experimentellen Wirtschaftswissenschaften, welche sich vorrangig auf das Gebiet der Verhaltensökonomie fokussiert hatten. Die Durchführung von ökonomischen Laborexperimenten bildete dabei einen zentralen Gegenstand im Arbeitsalltag dieser wirtschaftswissenschaftlich Forschenden. Die Anlage des Projekts zielte darauf ab, gemeinschaftlich zu 
untersuchen, welchen Einfluss Kultur ${ }^{1}$ auf die Ergebnisse von (v. a. spieltheoretischen) wirtschaftswissenschaftlichen Laborexperimenten ausübte. Wie dies erfolgen sollte, wurde zu Beginn des Projekts noch recht offen gehalten und erst im Zuge des Projekts selbst ausgehandelt.

In gemeinsamen Projektsitzungen galt es somit, Themen zu bestimmen, die für eine Versuchsanordnung im Labor geeignet waren, sowie aufbauend auf diesen Ideen Experimente zu konzipieren und diese im Labor umzusetzen. Die Experimente zielten darauf ab, zu ermitteln, ob die Einbeziehung kulturell aufgeladener Aspekte zu einer messbaren Veränderung der Ergebnisse im Labor führen würde. Auch die Auswertung der Ergebnisse der Experimente und das Verwerfen von Experimentserien wurde in gemeinsamen Sitzungen diskutiert. Im Rahmen des Projekts wurden drei Serien von Experimenten durchgeführt. Da die Experimente jedoch darauf ausgerichtet waren, im wirtschaftswissenschaftlichen Forschungslabor durchgeführt $\mathrm{zu}$ werden und auch als ökonomische Laborexperimente verstanden werden sollten, ergaben sich gewisse Grenzen der gleichberechtigten Einflussnahme auf die Konzeption der gemeinsamen Experimente.

Neben der gemeinsamen Projektfragestellung nach dem Einfluss von Kultur auf die Rahmung von Entscheidungssituationen im Labor wurde von Beginn an klar artikuliert, dass ich mich zugleich der Analyse des typischen Forschungshandelns der wirtschaftswissenschaftlichen ForscherInnen widmen würde. Der Projektzusammenhang bot dabei die entscheidende Grundlage für die Durchführung einer ethnographischen Studie. ${ }^{2}$ Dies stellte zugleich einen entscheidenden Vorteil für den Feldzugang dar, da dieser somit durch das Projekt selbst bereits hergestellt wurde. Die ProjektkollegInnen und auch die anderen Mitglieder der Forschungsabteilung begegneten mir freundlich, aufgeschlossen und gesprächsbereit. Diese projektbedingte Offenheit ist im Feld der verhaltensökonomischen Laborforschung jedoch nicht allgegenwärtig. Aufgrund der gängigen Vorwürfe gegenüber Laborexperimenten hinsichtlich ihrer Künstlichkeit und der damit einhergehenden geringen externen Validität der Ergebnisse sowie dem Boom des

\footnotetext{
${ }^{1}$ Kultur wurde dabei, orientiert am Programm der soziologischen Abteilung, als ,,kulturelle Konfigurationen“ (Hutter et al. 2010) definiert. Diese kulturellen Konfigurationen treten als leitende Interaktionsmuster in gemeinsamen Semantiken und Praktiken in Erscheinung und zeichnen sich durch phänomenspezifische raumzeitliche Ausdehnungen aus (ebd.).

${ }^{2}$ Zur weiteren Charakterisierung meiner Studie als Ethnographie siehe Abschnitt 4.1.2.
} 
Themas ,Nudging ${ }^{3}$ und der damit verbundenen Kritik, zeigen sich verhaltensökonomische ForscherInnen oft eher zurückhaltend, wenn es um die Erforschung ihrer wissenschaftlichen Praxis geht.

Ausgehend von einer Grundposition der verstehenden Soziologie und inspirierte durch ethnomethodologische Studien, stand für mich dabei von vornherein fest, dass eine reine Beobachtung und Beschreibung des Prozesses des Laborexperiments und der Arbeit der Laborforschenden nicht ausreichen würde, um diese zu erfassen. Nicht nur das Beobachten, sondern auch das Erlernen des typischen Forschungshandelns und der situationsangemessene Einsatz von Ethnomethoden der experimentellen Wirtschaftsforschenden und Labormitglieder ${ }^{4}$ bildete ein zentrales Ziel meines Forschungsprozesses. Dieser Lernprozess und seine Reflexion stellt eine zentrale Ressource für den hier dargelegten Forschungsprozess dar.

\section{Ablauf der Datenerhebung im Rahmen der Projektarbeit}

Während sich die verhaltensökonomischen ForscherInnen, mit denen ich zusammenarbeitete, auf die Auswertung der quantitativen Daten konzentrierten, war ich als qualitativ arbeitende Soziologin an Daten anderer Art interessiert. Im Rahmen der Projektarbeit zeigten sich nun unterschiedliche Verzahnungen der qualitativen und quantitativen Datenerhebung. Der nachfolgende Überblick über den Ablauf der Projektarbeit zeigt auf, welchen Einfluss meine Datenerhebung auf die Entwicklung des Projekts hatte und wie diese wiederum im Gegenzug durch das Projekt geprägt wurde.

Meine Kundgabe, dass ich gerne teilnehmende Beobachtungen von unterschiedlichen Laborexperimenten durchführen würde, stieß anfänglich auf ein gewisses Unverständnis. Im Selbstverständnis der Labormitglieder gab es im Labor nicht viel zu sehen, da die ProbandInnen ja schließlich die ganze Zeit nur am Computer sitzen würden. Trotz dieser Irritation über den Sinn meiner Beobachtungen willigte man ein. Bereits recht früh im Forschungsprozess begann ich mit der Durchführung von Interviews und Gesprächen. Anfangs wurden offene Interviews mit Mitgliedern des Laborteams und einzelnen ProbandInnen geführt. Aufbauend auf ersten Erkenntnissen und Hypothesen wurde von mir ein erster

\footnotetext{
${ }^{3}$ Hier verstanden als die Nutzbarmachung verhaltensökonomischer oder verhaltenspsychologischer Erkenntnisse und ihre Anwendung außerhalb der Wissenschaft im Bereich von Politik und Marketing. Siehe auch Abschnitt 2.2.3.

${ }^{4}$ Diese begriffliche Differenzierung ist notwendig, da die untersuchten experimentellen Wirtschaftsforschenden auch in dem untersuchten Labor forschten, jedoch nicht alle Labormitglieder Forschende der experimentellen Wirtschaftswissenschaften waren. Auch geschulte studentische Hilfskräfte und technische Mitarbeitende, die die Durchführung der Sessions betreuen, sind Labormitglieder.
} 
Leitfaden für die Interviews ausgearbeitet. TeilnehmerInnen wurden dabei kurz nach dem Abschluss des eigentlichen Experiments interviewt. Parallel wurden weitere Experimente teilnehmend beobachtet.

Im Rahmen der Projektarbeit wurde in gemeinsamen Sitzungen diskutiert, welche Experimente für die Projektfragestellung geeignet erschienen. Aufbauend auf diesen Diskussionen wurde in der (verhaltens-)ökonomischen Literatur nach Experimenten zum Thema Endowment-Effekt ${ }^{5}$ gesucht. Die entsprechenden Texte wurden diskutiert und in Anlehnung an ein klassisches Experiment in diesem Bereich wurde ein erstes Pilotexperiment mit dem Titel „Tassen und Pralinen"66 konzipiert. Die Schritte für die Umsetzung wurden im Labor geplant und vorbereitet. Dabei wurden zwei Sessions der Experimente im Labor durchgeführt - ein Kontrolltreatment und ein Versuchstreatment, welches durch einen kulturellen Einflussfaktor variiert wurde. ${ }^{7}$ Wie in der Durchführung von

${ }^{5}$ Der Endowment-Effekt (deutsch Besitztumseffekt) beschreibt einen in der verhaltensökonomischen Forschung viel untersuchten Effekt. Dieser besagt, dass Menschen einen höheren Verkaufspreis (WTA - Willingness to accept) für ein Gut oder eine Ware festlegen, welches sie besitzen, als sie bereit wären als Kaufpreis für ein solches Gut zu bezahlen (WTP - Willingness to pay) (Kahneman et al. 1990, 1991). Vereinfacht gesagt, geht der Endowment-Effekt davon aus, dass Menschen mehr Geld für den Verkauf von Dingen, die sie bereits besitzen erwarten, als sie selbst bereit wären für eben solche Dinge auszugeben. In der experimentellen Forschung ist dieser Effekt jedoch nicht unumstritten. Im Sinne der klassischen Ökonomie und dem rationalen Homo oeconomicus stellt dieses Verhalten eine Anomalie des Entscheidungsverhalten dar. Nach Charles Plott und Katrin Zeiler $(2005,2007)$ ist die Differenz zwischen Kauf- und Verkaufspreisen eine Konsequenz aus Verzerrungen, die sich durch die Durchführungen von Experimenten ergeben. In ihren Studien $(2005,2007)$ zeigen sie, basierend auf der Durchführung eigener Experimente, dass diese ,misconceptions“ und Abweichungen von einer rationalen Beurteilung des Wertes eines Gutes durch gezielte experimentelle Prozeduren ausgeschaltet werden können. Trotz der enormen Rezeption und Bekanntheit der Ergebnisse von Plott und Zeiler $(2005,2007)$ wurden diese in den folgenden Jahren jedoch durchaus wieder in Frage gestellt, insbesondere durch die Untersuchung von Isoni et al. (2011). Darauf reagierten Plott und Zeiler (2011) wiederum mit einer Replik, welche die Legitimität der Schlussfolgerungen des Aufsatzes von 2005 begründetet.

${ }^{6}$ Der Aufbau des Experiments und auch die Entscheidung für das Tauschobjekt der Tassen war dabei an einer klassischen Studie von Daniel Kahneman (1990) orientiert.

${ }^{7}$ In diesem Experiment sollte untersucht werden, ob sich ein signifikanter Unterschied in der Tauschbereitschaft von ProbandInnen zeigen würde, wenn in die Experimentsituation der kulturelle Aspekt eines Geschenks eingeführt werden würde. Die Versuchsgruppe erhielt dabei eine kleine Schachtel Pralinen, die als Geschenk des Universitäts-Labors markiert waren. Die Kontrollgruppe hingegen eine Tasse mit dem Universitätslogo. Als Tauschobjekt wurde beiden Gruppen das gleiche Objekt (ein Notizbuch mit Universitätslogo) angeboten. Verglichen wurde im Abschluss die Tauschbereitschaft der ProbandInnen der Versuchs- und Kontrollgruppe. 
Laborexperimenten üblich, wurden die Teilnehmenden am Ende des Experiments gebeten, am Computer einen kurzen Fragebogen auszufüllen. Dieser erfasste primär die sozio-demographischen Daten der ProbandInnen. Drei Freifelder boten die Gelegenheit, die Entscheidungshintergründe für die Wahlhandlungen im Experiment $\mathrm{zu}$ beschreiben und Lob und Kritik am Experiment zu äußern. Im Anschluss an diese Experimente führte ich gemeinsam mit dem Kollegen aus der Ökonomie zwei Gruppeninterviews mit jeweils sechs ProbandInnen durch. Das Experiment erbrachte aus wirtschaftswissenschaftlicher Perspektive keine signifikanten Ergebnisse und wurde aufgrund dessen verworfen. Nach diesem Experiment pausierte das Projekt für ein Jahr. Der wissenschaftliche Mitarbeiter der wirtschaftswissenschaftlichen Abteilung wechselte mit der Wiederaufnahme des Projekts.

Bei der Wiederaufnahme des Projekts wurde eine zweite Serie von Experimenten konzipiert, die sich ebenfalls im Bereich des Endowment-Effekts verortet lassen. Diese Experimentserie wurde intern als „Exklusivitätsexperiment“ bezeichnet. In Versuchs- und Kontrollgruppen wurde hier untersucht, ob einzelne Auswahlmöglichkeiten von Lotterien von ProbandInnen signifikant häufiger ausgewählt werden würden, wenn sie „exklusiv“ nur für einen Teil der ProbandInnen zugänglich sind. In der Vorbereitung dieser Experimente wurde in gemeinsamer Arbeit ein ausführlicher Fragebogen konzipiert, der im Rahmen der Experimente von allen ProbandInnen am Computerbildschirm ausgefüllt werden musste. Diesmal wurden 12 Sessions von Experimenten durchgeführt. Meine Beteiligung bei der Durchführung der Experimente war hier deutlich aktiver: Ich wurde dabei nicht nur stärker in das Verfassen der Instruktionen involviert, sondern auch in das Geschehen im Labor. Ich begrüßte die StudentInnen und wies sie auf die Verhaltensregeln im Labor hin. Unter Anleitung der anwesenden KollegInnen agierte ich als Mitglied des Laborteams. Meine Beobachterposition verschob sich durch die zunehmende Aktivität meiner eigenen Teilnahme und Involviertheit in die Prozesse des Laborgeschehens. Es wurden weiterhin Interviews mit ProbandInnen durchgeführt und bei 11 der 12 Sessions im Anschluss Interviewdaten erhoben. Die Interviews dienten dabei nicht nur meinem eigenen Forschungsinteresse, das Phänomen des Laborexperiments und seiner Rahmung zu entschlüsseln, sondern beinhalteten auch spezifische Fragen zu den jeweiligen Experimenten, an denen die ProbandInnen teilgenommen hatten. Wiederkehrende Aspekte aus diesen Interviews wurden in den gemeinsamen Projektsitzungen thematisiert und flossen in die Ausarbeitung der standardisierten Fragebögen ein, die gezielt nach der Verbindung von Spiel- und Spielertypen im Zusammenhang mit dem Verhalten in Experimenten fragten. Die Experimentserie „Exklusivitätsexperiment“ 
wurde verworfen, da sie für die ökonomischen ForscherInnen keine signifikanten und relevanten Ergebnisse erbrachte. Auch wenn sich hier quantitativ kein Zusammenhang nachweisen ließ, so flossen die Erkenntnisse der Interviews und Fragebögen doch in der Konzeption der nachfolgenden Serie von Experimenten ein.

Während im Rahmen der gemeinsamen Projektarbeit an der Planung und Konzeption der nächsten Serie von Experimenten gearbeitet wurde, bereitete ich parallel die erste Erhebung von Videodaten vor, um den Ablauf des Laborgeschehens detailliert in seiner Natürlichkeit zu erfassen. Zum Zweck der Datenerhebung wurden drei Sessions eines Experiments zum Thema Mindestlohn durchgeführt, welches ich als teilnehmende Beobachterin zuvor mehrere Male beobachtet hatte. In allen drei Sessions wurden im Anschluss Interviews geführt.

In der dritten Serie von Experimenten des Brückenprojekts sollte bestimmt werden, ob es die Bewertung eines Objekts verändert, wenn man seine Besitzrechte in einem Spiel gewinnt. Es wurden dabei unterschiedliche Spielformate als Teil des Ablaufes eingesetzt, um zu vergleichen, ob diese - bei sonst gleichbleibenden Versuchsanordnungen - zu einer Änderung des Entscheidungsverhaltens der ProbandInnen beitrugen. Diese Versuchsreihe wird im Folgenden als „Endowment-Effekt USB-Stick“ bezeichnet, da ein USB-Stick das Objekt war, dessen Bewertung in diesem Experiment untersucht wurde. In der Planung für diese Experimente wurden in der Projektgruppe ein Fragebogen ausgearbeitet und Hintergründe der Festlegung der Kauf- und Verkaufspreise erfragt. Zudem befasste sich die Projektgruppe intensiv mit der Erarbeitung der Instruktionen und der Planung der Abläufe der Sessions. ${ }^{8}$ Im Rahmen des Projekts wurden 19 Sessions durchgeführt und in 10 der 19 Durchgänge Interviews geführt. Verstärkt wurden dabei Fragen zum Verständnis und Vorgehen in spezifischen Entscheidungssituationen des Experiments gestellt. An der Durchführung dieser Experimente im Labor war ich maßgeblich beteiligt und bis auf einen krankheitsbedingten Ausfall habe ich selbst alle Experimente im Labor angeleitet.

\footnotetext{
${ }^{8}$ Die Versuchsanordnung dieser Experimentserie war dabei stark an der Untersuchung von Plott und Zeiler (2005) orientiert. Im Baseline Treatment (der Kontrollgruppe) wurde dabei angestrebt, die Studie so weit wie möglich zu replizieren. Orientiert an Plott und Zeiler (2005) durchliefen die ProbandInnen eine Reihe von Runden, in denen sie als Käufer oder Verkäufer den Preis für Lotterietickets festlegen mussten. Es wurde hier ein spezifischer ökonomischer Mechanismus - der BDM-Mechanismus (siehe 5.3.6) - eingesetzt, um mit den ProbandInnen rationales Entscheiden bei der Preisfestlegung zu trainieren. In einer letzten Spielrunde sollten die ProbandInnen, je nach zugewiesener Rolle, den Kauf- bzw. Verkaufspreis für einen USBStick bestimmen.
} 
In den nachträglichen Projektsitzungen wurden die Ergebnisse der Experimente intensiv diskutiert. In späteren Phasen der Auswertung wurde versucht, die quantitativen Ergebnisse, die aus der Datenerhebung im Labor hervorgingen, mit den Fragebogendaten und Interviews zu triangulieren. Aufbauend auf dem Datenmaterial der Fragebögen und Interviews wurden Hypothesen generiert und an dem quantitativen Material ${ }^{9}$ hinsichtlich ihrer Signifikanz getestet.

Parallel zu diesen Diskussionen wurde eine letzte Datenerhebung im Labor vorgenommen. Es handelte sich dabei um vier Experimente, die auf Grundlage meiner Auseinandersetzung mit der Methode des qualitativen Experiments nach Gerhard Kleining (1986) konzipiert wurden. Inhaltlich wurde dabei das Grundexperiment (Baseline Treatment) der USB-Stick-Serie verwendet. Diese Experimente wurden von mir geplant, vorbereitet und im Labor durchgeführt. Zugleich wurden sie mittels Video aufgezeichnet. Im Anschluss an die Sitzungen wurden jeweils Interviews geführt. Einige Ergebnisse der qualitativen Experimente wurden in der Projektgruppe diskutiert und flossen selbst in die abschließende Vorstellung der Projektergebnisse ein.

Diese ersten einleitenden Bemerkungen zur Chronologie des Forschungsprozesses zielten darauf ab, eine gewisse Transparenz für das Wechselspiel der Projektarbeit und der Herausbildung meines Forschungsprozesses zu schaffen. In einem nächsten Schritt soll nun das Forschungsdesign spezifiziert werden, welches das Vorgehen der Arbeit prägte.

\subsubsection{Einordnung der eigenen Studie als Ethnographie}

Ein zentraler Anspruch der verstehenden Soziologie besteht darin, die altägliche Welt der Handelnden zum Primat der empirischen Untersuchung zu machen. Dazu gibt es verschiedene methodische Wege. Das Vorgehen der Ethnographie besteht darin, an den alltäglichen und typischen Handlungsvollzügen der Handelnden teilzunehmen und diese zu beobachten. Auf diese Weise soll es gelingen, sich mit der Lebenswelt der Beobachteten vertraut zu machen und ihre Handlungen nachvollziehen und beschreiben zu können. Häufig wird die Ethnographie auch zugleich allgemeiner als Feldforschung bezeichnet (Breidenstein et al. 2015; Girtler 2001).

Aus etymologischer Perspektive lässt sich der Begriff der Ethnographie als die Beschreibung von Völkern verstehen. Das Vorgehen der Ethnographie geht auf

\footnotetext{
${ }^{9}$ Mit „quantitativem Material“ sind hier die Ergebnisse gemeint, die sich im ersten Teil der Experimente durch die Kauf- und Verkaufsentscheidungen der ProbandInnen ergaben. Dieser Begriff ist hier etwas zweideutig, da auch die Ergebnisse der Fragebögen quantitative Ergebnisse sind. Siehe dazu auch Abschnitte 4.2.1.5 und 4.2.1.6.
} 
den Begründer der Ethnologie Bronislaw Malinowski zurück. Dieser etablierte die teilnehmende Beobachtung als Standardmethode der ethnologischen Forschung. Malinowski postulierte in seinen Studien die methodologische Forderung, dass Forschung nicht vom ,Liegestuhl der Veranda“ (Malinowski 1973: 128) ausgehend mittels Sekundärdaten erfolgen sollte, sondern sich vielmehr aktiv und intensiv dem Mitvollzug des Alltagslebens, der Rituale und Gebräuche der untersuchten Gruppe widmen müsse. Malinowskis methodische Postulate spezifizierten dabei drei Grundbedingungen ethnographischer Forschung, die bis heute Relevanz besitzen: die längere Anwesenheit im Forschungsfeld, die Einnahme der Binnenperspektive der beforschten Gruppe und die Erarbeitung eines Verstehens des Fremden und Unvertrauten (vgl. Breidenstein et al. 2015: 18).

Klassische Studien im Bereich der Kulturanthropologie und der Ethnologie befassen sich primär mit der Untersuchung außereuropäischer Kulturen und indigener Volksstämme (Malinowski et al. 1979). Soziologische Untersuchungen, die das Vorgehen der Ethnographie verwenden, beobachten im Gegensatz dazu meist spezifische Gruppen ihrer eigenen Kultur (Hirschauer \& Amann 1997). Die fruchtbare Anwendung der ethnographischen Methode für die soziologische Forschung zeigt Anne Honer auf. Sie bezeichnete diese Studien, die sich der Untersuchung der uns umgebenden Welt widmen, dabei als „Beschreibung von kleinen Lebenswelten“ (Honer 2000). Im Folgenden wird nun dargestellt, dass meine Studie von der Idee geleitet wurde, die „,kleine Lebenswelt“ der verhaltensökonomischen ForscherInnen und ihrer Arbeit im Labor zu untersuchen. ${ }^{10}$ In der Auseinandersetzung mit dem Forschungsfeld ergab sich dabei schrittweise eine Spezifizierung des Forschungsgegenstands. Im Zuge der Feldarbeit wurden verschiedene Methoden der Datenerhebung genutzt. Diese wurden dabei eingesetzt, um verschiedene Aspekte des Beobachtungsfeldes genauer zu betrachten. Der spezifische Anspruch und Wert, der den jeweiligen Verfahren im Forschungsprozess beigemessen wurde, wird im Abschnitt 4.1.3 thematisiert. In einem ersten Schritt wird nun jedoch dargestellt, wodurch die Einordnung meiner Studie als eine soziologische Ethnographie zu begründen ist.

\section{Einordnung der eigenen Studie als soziologische Ethnographie}

Sowohl das allgemeine Vorgehen als auch die vielfältigen Datensorten und Erhebungsmöglichkeiten, die genutzt wurden, um eine intensive Auseinandersetzung

\footnotetext{
${ }^{10}$ Eine Inspiration stellte dabei der Aufsatz „Life among the econ“ des Ökonomen Axel Leijonhufvud (1973) dar, in welchem er sich einer Beschreibung des „Stammes“ der wirtschaftswissenschaftlichen ForscherInnen zuwandte.
} 
mit dem Feld zu bewerkstelligen, charakterisieren meine Untersuchung als soziologische Ethnographie. Im Rahmen der gut vierjährigen Projektlaufzeit arbeitete ich intensiv mit den wirtschaftswissenschaftlichen ForscherInnen zusammen und verbrachte zum Zwecke der Ethnographie mehrere Monate in ihrer Abteilung. Dort nahm ich an ihrem Abteilungsalltag, gemeinschaftlichen Mittagessen und Kaffeepausen teil. Ich besuchte ihre Kolloquien und verfolgte hier, wie sie ihre eigene Forschung in der Peergroup darstellten, kritisierten und legitimierten. Während meines Aufenthaltes in der ökonomischen Abteilung bezog ich ein gemeinsames Büro mit einem jungen Post-Doktoranden und später, nach einem räumlichen Wechsel, mit einem jungen wissenschaftlichen Mitarbeiter. Zudem wurde ich aktiv an der Konzeption und praktischen Vorbereitung verschiedener Experimente beteiligt. Ein wichtiger Aspekt war hier beispielsweise die Erstellung der schriftlichen Instruktionen für die unterschiedlichen Experimente. Eine entscheidende Vertiefung meines ethnographischen Feldwissens wurde durch meine aktive Teilnahme an der Durchführung der Experimente ermöglicht. Auf diese Weise gelang es mir, spezifisches Wissen über den praktischen Vollzug der Experimente und die gängigen Praktiken und situativen Lösungsmethoden der ExperimentatorInnen zu erlernen. Im Verlauf der Forschungsarbeit bildete sich dabei sukzessive eine Fokussierung meines analytischen Interesses auf den typischen Vollzug der Experimente und die Prozesse der Rahmenbildung heraus, die die beobachteten Laborexperimente in ihrer spezifischen Eigengesetzlichkeit hervorbrachten.

In ethnographischen Untersuchungen ist es üblich, sich offen einem Forschungsfeld zu nähern und sich erst im Forschungsprozess selbst sukzessiv auf bestimmte Aspekte zu fokussieren. Dieses Vorgehen einer schrittweisen Fokussierung ist auch ein charakteristisches Moment meines eigenen Forschungsprozesses. Meine spezifische Art der ethnographischen Untersuchung weist dabei eine gewisse Nähe zur „,fokussierten Ethnographie“ (Knoblauch 2001) auf, ohne selbst jedoch als solche eingeordnet werden zu können. Zur Charakterisierung der von mir vorgenommenen ethnographischen Untersuchung ist es hilfreich, dies etwas genauer zu explizieren.

Die fokussierte Ethnographie wird durch zwei zentrale Aspekte charakterisiert. Zum einen macht sie, wie in der soziologischen Ethnographie üblich, ihre eigene Gesellschaft und Kultur zum Gegenstand ihrer Untersuchung. Dies ermöglicht es den Forschenden, auf ihr eigenes (Alltags)wissen als Ressource zurückzugreifen, um den relevanten Kontext der untersuchten Situationen zu verstehen. Dieses ethnographische Kontextwissen der Forschenden eröffnet zugleich die Möglichkeit, sich in ihren Analysen auf spezifische Details zu fokussieren. Der entscheidende Schritt für diese Fokussierung und zugleich das zweite Charakteristikum 
der fokussierten Ethnographie ist der Einsatz audiovisueller Aufzeichnungsgeräte zur Datenerhebung. Im Unterschied zu ,,zeitintensiven“ klassischen ethnographischen Studien, bei denen die Forschenden Monate oder sogar Jahre im Feld verbringen und Feldnotizen sammeln, sind die Videodaten, auf denen die fokussierte Ethnographie ihre Analysen gründet, besonders ,datenintensiv“ (Knoblauch 2001: 129). Datenintensiv meint hier, dass es die im Feld aufgezeichneten Videodaten ermöglichen, die vorgefundenen Interaktionen in ihrem natürlichen Vollzug zu konservieren und so bis ins feinste Detail zu rekonstruieren. Zugleich können neben den verbalsprachlichen Äußerungen auch die weiteren multimodalen Aspekte der Kommunikationssituationen in der Videoaufzeichnung erfasst werden, wie Mimik und Gestik der Interaktionspartner oder räumlich-materielle Aspekte. Bei ihren Feldaufenthalten produzieren die Forschenden dabei meist mehrere Stunden Videomaterial, welches nachfolgend analysiert wird. Aufenthalte der Forschenden im Feld wechseln sich dabei meist mit den Analysen der Daten ab. Dieses für die fokussierte Ethnographie typische Untersuchungsverfahren, welches Videoanalysen natürlicher Interaktionssituationen zum Brennpunkt seiner Analyse macht, wird als ,Videographie“ (Schnettler \& Knoblauch 2009; Tuma et al. 2013) bezeichnet. Die Videographie kann somit als die Methode der fokussierten Ethnographie bezeichnet werden. Durch die Videographie eröffnet sich zugleich auch ein anderer Blickwinkel auf die Performanz und Performativität des Interaktionsgeschehens. Mittels eines sequenzanalytischen Vorgehens werden dabei die in den Videodaten konservierten natürlich Interaktions- und Handlungsprozesse in ihrem Vollzug betrachtet und analysiert. Das analytische Vorgehen der Videographie reichert diese Sequenzanalyse jedoch durch das ethnographische Wissen der Forschenden an. Diese Verbindung von Sequenzanalyse und Ethnographie verweist zugleich darauf, dass das Prozesshafte der Performanz häufig nicht im Situativen aufgeht, sondern zugleich Institutionalisierungen mit einbezieht (vgl. Schnettler et al. 2007: 21).

Meine Orientierung an charakteristischen Momenten der fokussierten Ethnographie besteht eben in diesem methodischen Vorgehen der Videographie. Dieses sieht in der Analyse von Videosequenzen die Chance, den natürlichen Vollzug von Interaktionsphänomenen ,einzufrieren“ und so dem komplexen Zusammenspiel von sprachlichen, körperlichen und materiellen Aspekten der Untersuchungssituationen gerecht zu werden. Wie aus der dargestellten konzeptionellen Perspektive meiner eigenen Forschungsarbeit (Kapitel 3) hervorgeht, wird eben dieser Vielschichtigkeit von Interaktionssituationen ein zentraler Stellenwert beigemessen, um die Prozesse der Rahmenbildung und -verankerung im Experimentallabor zu untersuchen. Die Datenintensität, die von Videoaufnahmen ausgeht, kann 
jedoch nur durch das ethnographische Wissen entschlüsselt werden. Zur Analyse und Interpretation der Videodaten wird somit maßgelblich auf die Kenntnisse des Forschenden über die relevanten Kontextelemente und Wissenshintergründe zurückgriffen. Im Unterschied zur fokussierten Ethnographie richtet sich mein Forschungsinteresse jedoch nicht auf ein Phänomen der Alltagswelt, sondern auf einen Forschungsgegenstand, der mir zu Beginn meiner Untersuchung ,fremd war. Wissenssoziologische Untersuchungen, die sich mit spezifischen Wissenschaftsbereichen befassen, die nicht ihrer eigenen Ausbildung entsprechen, stehen immer vor dem Problem, sich das relevante ethnographische Wissen durch eine Auseinandersetzung im dem Feld aneignen $\mathrm{zu}$ müssen. Das ethnographische Wissen, welches in meinem Fall zur Betrachtung des Untersuchungsphänomens benötigt wird, wurde in der Projektarbeit und in der Auseinandersetzung mit dem Feld gewonnen. Wie nachfolgend dargestellt, wurden dafür verschiedene Verfahren der Datenerhebung genutzt. Aus diesem Grund muss meine Untersuchung sowohl als datenintensiv als auch als zeitintensiv charakterisiert werden.

Die Datenintensität, die das Videomaterial bietet, ist dabei für die Analyse von entscheidender Bedeutung. Die soziologische Gattungsanalyse, welche von mir durchgeführt wird, ist ein empirisches wissenssoziologisches Verfahren, welches sich selbst als datengeleitet versteht und aus diesem Grund auf der Analyse empirisch beobachtbarer Daten fußt. Durch die Videodaten wird es dabei möglich, die situative Hervorbringung der Rahmung des Laborexperiments zu betrachten und die weiteren ethnographischen Erkenntnisse und Daten mit diesem Prozess zu triangulieren. Das zentrale Bezugssystem stellt dabei der Ablauf dar, den die Videodaten konservieren. Die Kamerapositionierung und die räumlich-visuellen Beschränkungen durch die Trennwände begrenzen jedoch auch den Detailgrad der Videoaufzeichnungen.

\section{Meine Feldrolle und ihre Entwicklung}

Die teilnehmende Beobachtung gilt als die klassische Datenerhebungsmethode in der ethnographischen Forschung (Girtler 2001; Knoblauch 2014). Sie zielt darauf $a b$, dass der Forschende ein Verständnis für die beforschte Gruppe und ihren Alltag entwickelt, indem er einen längeren Zeitraum mit ihnen verbringt, an ihren (täglichen) Ritualen und Routinen teilhat und diese zugleich wissenschaftlich reflektiert und beobachtet. Die Position oder Feldrolle, die ein teilnehmender Beobachter oder eine Beobachterin einnimmt, unterscheidet sich dabei deutlich von der eines außenstehenden Beobachters. ${ }^{11}$ Der Forschende ist dabei kein

\footnotetext{
${ }^{11}$ Diese Form des Beobachtens wird in charakteristischer Form in Marktforschungsinstituten oder auch bei Polizeiverhören genutzt, in denen Einwegspiegel eingesetzt werden. Diese
} 
Außenstehender. Es wird angestrebt, dass er eine Rolle im Feld einnimmt und von der Gruppe, die er beobachtet, nicht als störend oder irritierend wahrgenommen wird. Die Feldrolle verändert sich dabei üblicherweise im Rahmen des Feldkontaktes und es können unterschiedliche Grade der Teilnahme im Feld unterschieden werden. So unterscheidet Hitzler hinsichtlich der Involviertheit des Forschenden in das Feld beispielsweise zwischen „Old School“ und „New School“ (Hitzler et al. 2016). Neuere Formen der ethnographischen Feldforschung sind dabei üblicherweise durch eine stärkere Involvierung der Forschenden in das Feld charakterisiert. Da die Feldrolle des Forschenden somit ein zentrales Kriterium ist, soll die Entwicklung meiner eigenen Feldrolle im Folgenden etwas ausführlicher dargestellt werden. ${ }^{12}$

$\mathrm{Zu}$ Beginn des Projekts war mir das Feld der wirtschaftswissenschaftlichen Laborforschung weitestgehend unbekannt. Eine erste Annäherung an das Feld begann durch die teilnehmende Beobachtung unterschiedlicher Experimente. Die Experimente fanden dabei vor allem im wirtschaftswissenschaftlichen Labor einer Berliner Universität statt. Ergänzend wurden auch einzelne Beobachtungen in einem anderen Experimentallabor in Berlin getätigt. ${ }^{13}$ Im Rahmen dieser ersten Feldkontakte wurde die Chance genutzt, mit den ExperimentatorInnen zu sprechen, die die Experimente begleiteten. Diese ersten Beobachtungen dienten vorrangig dazu, den allgemeinen Ablauf der Laborexperimente zu erfassen. Im Zuge der ersten Reihe von Experimenten, die im Rahmen des Projekts durchgeführt wurden, erfüllte ich einfache Aufgaben in der Vorbereitung der Experimente: Im Labor überprüfte ich beispielsweise, dass an allen Arbeitsplätzen vor Beginn des Experiments Instruktionen und Stifte bereitlagen. Im Rahmen der Experimente selbst nahm ich jedoch die Position einer teilnehmenden Beobachterin ein und war nicht aktiv in die Durchführung eingebunden.

Wie oben beschrieben, eröffnete sich mir im Rahmen des Projektzusammenhangs die Möglichkeit, das situativ angemessene Handeln eines Labormitglieds und die damit verbundenen praktischen Techniken und Methoden der adäquaten Problemlösung zu erlernen. Diese Phase markierte den Übergang von der primär teilnehmenden Beobachtung zur beobachtenden Teilnahme. Es erscheint mir

ermöglichen es den Personen hinter dem Spiegel, das Geschehen zu beobachten, ohne selbst als Teil der Szenerie in Erscheinung zu treten.

${ }^{12}$ Teile dieser Darstellung der Entwicklung meiner Feldrolle wurden aus meinem Artikel Haus (2018) übernommen.

${ }^{13}$ Im Unterschied zu den Experimenten im Experimentallabor an der Universität, die alle in deutscher Sprache durchgeführt wurden, vollzog sich im zweiten Labor der gesamte Prozess des Experiments - von der Begrüßung der ProbandInnen bis zu ihrer Bezahlung - in englischer Sprache. 
jedoch notwendig, diesen Prozess genauer zu reflektieren und zugleich darauf zu verweisen, welche Probleme sich durch diesen Rollenwechsel für mich als Forschende, aber auch für die Beforschten ergaben.

In einer fortgeschrittenen Phase meines Feldaufenthalts wurde ich nicht nur in die Vorbereitung der Experimente bezüglich des Schreibens der Instruktionen und das Ausstatten der Arbeitsplätze mit dem nötigen Equipment involviert. Aufgrund eines forschungspraktischen Zufalls wurde ich selbst als Experimentatorin geschult. Der Grund dafür bestand darin, dass sich im Rahmen des Projekts ein personeller Wechsel auf der ökonomischen Seite des Projekts ergab und der neue wissenschaftliche Mitarbeiter kein deutscher Muttersprachler war. Da die Experimente im genutzten Labor jedoch üblicherweise in deutscher Sprache durchgeführt werden, wurde ich gebeten, die Experimente anzuleiten. Wie an anderer Stelle ${ }^{14}$ ausgeführt, sind die Forschenden im Rahmen von ökonomischen Experimenten bestrebt, die Erhebungssituation so weit wie möglich zu standardisieren und potenzielle Einflussfaktoren im Rahmen der Erhebungssituation konstant zu halten. Dies bezieht sich auch auf den Einsatz der ExperimentatorInnen. Um diesen potenziellen Störfaktor in den Daten konstant zu halten, werden im untersuchten Experimentallabor bevorzugt die gleichen ExperimentatorInnen für die Durchführung unterschiedlicher Sessions im Rahmen einer Serie von Experimenten eingesetzt. Dies bedingte, dass ich nicht nur eine Session eines Experiments durchführte, sondern gleich eine ganze Reihe von Erhebungen einer Experimentserie.

Schritt für Schritt erlernte ich dabei die nötigen Kompetenzen für das Agieren im Labor. Zu Beginn bestand meine Aufgabe lediglich darin, die Teilnehmenden zu begrüßen und ihnen die drei „Goldenen Regeln“ für das Verhalten im Labor mitzuteilen. ${ }^{15}$ Nachfolgend erlernte ich, die Informationen, die auf dem Übersichtsbildschirm, welcher am Arbeitsplatz der Labormitglieder den Prozess des Experiments abbildete, zu deuten und zu entscheiden, wann es Zeit war in Aktion zu treten. Im Rahmen meines Trainings stand mir dabei jeweils ein erfahrenes Labormitglied zur Seite, welches mich darauf hinwies, welche Handlungen zu welcher Zeit notwendig und angemessen waren. Es galt nun zunehmend den Ansprüchen gerecht zu werden, die von Seiten des Feldes an ein kompetentes Labormitglied gestellt wurden.

\footnotetext{
${ }^{14}$ Im Abschnitt 2.1 wurde dargestellt, dass das Konstanthalten der Randbedingungen ein zentrales Moment der Methodologie sozialwissenschaftlicher Experimente darstellt.

${ }^{15}$ Die ProbandInnen werden in dieser Phase darüber informiert, dass es ihnen untersagt ist, miteinander zu kommunizieren, dass sie Fragen bitte per Handzeichen anzeigen sollen und dass es verboten ist, eigene technische Geräte im Labor zu nutzen. Siehe dazu auch Abschnitt 5.3.3.
} 
Als problematisch erwies es sich für mich, die zeitlich Veraktung der Aktivitäten richtig einzuschätzen. Der enge Zusammenhang zwischen dem Handeln der ProbandInnen, dem Eingreifen der Labormitglieder und dem störungsfreien Arbeiten der technischen Software war mir anfänglich nicht bewusst. In einer Sitzung führte dies zu einem krisenhaften Zwischenfall. ${ }^{16}$ Die Konsequenz meines verspäteten Eingreifens bestand dabei im Absturz der Software. Da alle Computer im Labor in einem internen Netzwerk miteinander verbunden sind und die Abläufe rundenbasiert gesteuert werden, wurde es aus diesem Grund zugleich auch für alle anderen ProbandInnen unmöglich, mit dem Experiment fortzufahren. Die Komplexität der Situation zu überblicken und das eigene Handeln entsprechend zu koordinieren, erforderte Übung. Da diese „Übungen“ jedoch stets in realen Datenerhebungssituationen erfolgten, hatten Fehler zugleich folgenreiche Konsequenzen. Da die relevante Entscheidung, die mit dieser Versuchsanordnung erhoben werden sollte, erst nach diesem Zwischenfall erfolgt wäre, wurden die Daten des gesamten Experiments damit für die Analyse unbrauchbar. Mein auf mangelnder Praxis beruhendes „Fehlverhalten“ wurde somit zugleich zum Problem für meinen routiniert agierenden Projektkollegen. Die daraus resultierenden Spannungen im kollegialen Verhältnis konnten in der nachfolgenden Problematisierung dieses Sachverhalts jedoch geklärt werden. Wir verständigten uns darauf, dass die Ursache dieses Vorfalls zugleich maßgeblich in der technischen Konzeption des Experiments lag, welche entsprechend verändert werden konnte.

Die weitere Arbeit im Feld und die steigende Anzahl der durchgeführten Experimente erlaubten es mir, zunehmend die notwendigen Kompetenzen zu erwerben und situativ angemessen zu agieren. In späteren Phasen der Erhebung im Labor wurde mir aus diesem Grund das Vertrauen entgegengebracht, die Experimente auch allein durchzuführen. Jedoch waren auch in diesen Fällen stets weitere erfahrene Labormitglieder in erreichbarer Nähe, sodass bei größeren Problemen, wie technischen Zwischenfällen, jemand zu meiner Unterstützung hinzugezogen werden konnte. Diese Schulung als Experimentatorin ermöglichte auch, dass ich in einer späteren Projektphase - parallel zu den Datenerhebungen, die unserer gemeinsamen Projektarbeit dienten - eigene Experimente im Labor durchführen konnte.

\footnotetext{
${ }^{16}$ Meine Aufgabe wäre es hier gewesen, zu kontrollieren, dass an zwei zusammengehörigen Arbeitsplätzen im Labor eine übereinstimmende Eingabe getätigt wurde und dass dies in einem festgelegten Zeitrahmen erfolgte. Die beteiligten ProbandInnen gaben jedoch wiederholt Werte ein, die nicht die gewünschte Übereinstimmung zeigten. Als der dafür vorgesehene Zeitrahmen verstrichen war, führte dies zu einer Fehlermeldung im Programm, die den besagten Absturz der Software auslöste.
} 
Der Hintergrund für die Darstellung dieser empirischen Episode liegt darin, zu reflektieren, welche Konsequenzen sich durch die verstärkte Einbindung eines Feldforschenden für den Forschenden und das Feld selbst ergeben. Der ethnomethodolgischen Forderung der „Studies of work“ (Garfinkel 1986), die ,embodied practices“ (Bergmann 2006: 395) analysieren und explizieren zu können, kann nur entsprochen werden, wenn sich Forschende selbst aktiv mit der relevanten Praxis des Feldes auseinandersetzen und diese erlernen. Eine weite Herausausforderung, die sich insbesondere bei ethnographischen Studien ,fremder' Kulturen ergibt, ist die Herausforderung, die sinnliche Wahrnehmung der Beforschten und ihre Relevanz für den Interaktionsvollzug zu verstehen und zu erlernen (Meyer 2017: 146). Nimmt man im Feld eine Rolle ein, die auch für die dort agierenden eine maßgebliche Relevanz besitzt, eröffnet sich die Chance, die Sensibilität gegenüber den ablaufenden Prozessen in entscheidendem Maße zu steigern. Der Forschende selbst steht damit jedoch zugleich vor der Herausforderung, den Ansprüchen des Feldes gerecht zu werden. Fehler, die sich aus Unkenntnis oder Mangel an bisheriger praktischer Erfahrung ergeben, können damit zur Belastung für den Forschenden selbst und das Feld der „Einheimischen“ werden. Aus diesem Grund gilt es durchaus kritisch zu reflektieren, ob und inwiefern eine Involvierung eines fachfremden Forschenden in der jeweiligen empirischen Situation möglich und ethisch vertretbar ist.

\section{In-vivo-Kodes}

Die Aufnahme feldspezifischer Begriffe in die Analyse erfolgt in der Grounded Theory durch die Verwendung von In-vivo-Kodes (Strauss \& Corbin 1996: 25 ff.). In-vivo-Kodes greifen typische Begriffe des empirischen Feldes auf: „Sie haben häufig den Vorteil, Charakteristika des Gegenstandes im Idiom des Feldes (,authentisch“) zu fassen“ (Breuer 2009: 78). Markante Begriffe, typische Ausdrucksoder Redeweise, die sich im empirischen Material wiederkehrend zeigen, werden dabei direkt übernommen und in einen Kode überführt. Die In-vivo-Kodes bezeichnen dabei jedoch nicht mehr ein einzelnes empirisches Phänomen, sondern repräsentieren ein größeres Konzept, das sich an verschiedenen Stellen im empirischen Material zeigt. Gerade in der Analyse der Audiodaten der Interviews und der Projektsitzungen erweist sich die Aufnahme von In-vivo-Codes als sehr fruchtbar. Vielfältig werden hier typische Begriffe verwendet, die zugleich auf ganz spezifische Wissenshintergründe und Annahmen anspielen und auf diese verweisen. Die Kommunikationssituation im Labor selbst ist von der spezifischen Nutzung und Bedeutung von Feldbegriffen geprägt; so fußt beispielsweise die spezifische Bedeutung und das Verständnis des Kommunikationsverbots auf 
dem Akteursmodell der Forschenden. Auch Begriffe des Entscheidens, der Entscheidungssituation oder des Entscheidungsverhaltens sowie der Rationalität und dem rationalen Verhalten werden in ihrer feldspezifischen Nutzung in der Analyse beibehalten. Sogar der Begriff des Experiments selbst ist als Feldbegriff zu verstehen, da im vorliegenden Fall eine ganz spezifische Form des wirtschaftswissenschaftlichen Experiments bezeichnet wird und dabei konkreter häufig (sowohl von den ProbandInnen als auch von den Labormitgliedern) nur ein spezifischer Ausschnitt des sozialen Phänomens gemeint ist, der sich auf die Datenerhebung im Labor bezieht. Im Rahmen der vorliegenden Arbeit werden gezielt Feldbegriffe und In-vivo-Kodes eingesetzt, um eine authentische und dichte Beschreibung der ablaufenden Prozesse zu ermöglichen. Dabei wird es bewusst unterlassen, diese Begriffe selbst aus einer soziologischen Perspektive heraus hinsichtlich ihrer , legitimen Anwendung' zu reflektieren oder zu relativieren.

Bezüglich der nachfolgend diskutierten Frage der Reaktivität eingesetzter Verfahren der Datenerhebung ist der Grad der Involviertheit des Forschers in das beobachtete Feld ebenfalls ein relevanter Aspekt. Wie das oben aufgeführte Beispiel aufzeigt, führte der Zwischenfall in dieser Situation zu einer enormen Reaktanz, da die geplante Datenerhebung durch mich zentral beeinflusst wurde. Zugleich konnten jedoch auch in dieser Situation relevante Informationen über den Umgang und die situative Lösung von Krisensituationen gesammelt werden. Diese weisen durchaus Parallelen zum Management anderer krisenhafter Situationen auf, die im Rahmen der Feldarbeit beobachtet werden konnten.

\subsubsection{Die experimentelle Validierung durch das qualitative Experiment}

In der nachfolgenden Analyse wird die Gattungsanalyse als wissenssoziologisches Analyseverfahren genutzt, das es ermöglicht, die unterschiedlichen Datensorten $\mathrm{zu}$ triangulieren und so gewinnbringend in den Analyseprozess zu integrieren. Auf diese Weise wird herausgearbeitet, mittels welcher kommunikativer Handlungen die spezifische Laborumwelt hervorgebracht wird, die aus verhaltensökonomischer Perspektive rationales Entscheiden beobachtbar und messbar macht. Ausgehend von einer Perspektive der Forschenden und Labormitglieder wird dabei rekonstruiert, wie die Handlungsspielräume der ProbandInnen begrenzt und Verhaltenserwartungen ihnen gegenüber produziert werden und so, Schritt für Schritt im Zuge der Durchführung der Experimente, das spezifische Umfeld des ökonomischen Laboratops entsteht. 
Wie weiter oben ausgeführt, wurden im Forschungsprozess unterschiedliche Datenerhebungsmethoden verwendet, um sich dem Forschungsgegenstand schrittweise explorativ anzunähern. Der Anspruch der Gegenstandsadäquanz und des „unique adequacy requirements“ (Garfinkel \& Wieder 1992) war, wie bereits ausgeführt, ein zentraler Motor der Suchbewegung und des Forschungsprozesses. Wiederholt stellte sich im Rahmen meiner Datenerhebung die Frage, ob es möglich sei, dichter an das interessierende Phänomen der Hervorbringung der typischen Rahmung im ökonomischen Labor heranzukommen, ohne die natürliche Situation des Laborexperiments so weit zu verändern, dass das beobachtete Phänomen nicht mehr dem interessierenden Forschungsgenstand entsprochen hätte. Aufgrund meiner eigenen Feldrolle war es mir dabei möglich, mir vielfältiges Wissen über die Durchführung von ökonomischen Laborexperimenten anzueignen. Zudem wurden einige Hypothesen und Ideen durch die Beobachtungen und Interviews mit den Teilnehmenden gesammelt. Ob das Framing der ProbandInnen sich jedoch an den gleichen Aspekten orientierte, wie jene, die von den Forschenden und Labormitgliedern gezielt induziert wurden und welche Beziehung und Gewichtungen zwischen den verschiedenen Aspekten in der kommunikativen Konstruktion im Labor wirkten, war weiterhin nicht eindeutig zu bestimmen.

In meiner Auseinandersetzung mit den methodischen Grundlagen des Experiments und der Suche nach einer gegenstandsangemessenen Methode für mein Forschungsvorhaben stieß ich auf die in Vergessenheit geratene Methode des „qualitativen Experiments" nach Gerhard Kleining (1986), die vielen meiner forschungspraktischen Bedürfnisse zu entsprechen schien. ${ }^{17}$ Im Anschluss an die drei Ebenen der Analyse, die sich an der Gattungsanalyse orientieren, wird anhand einer eigenen kurzen Serie von qualitativen Experimenten illustriert, wie dieses Verfahren zur experimentellen Validierung der eigenen qualitativen Forschungsergebnisse herangezogen werden konnte. Für ein besseres Verständnis des Verfahrens werden im Folgenden einige grundlegende Aspekte des qualitativen Experiments eingeführt.

Bereits in seinem Aufsatz von 1986 verwies Kleining darauf, welches Potenzial die Wiederbelebung dieser Forschungsmethode besitzt. Doch scheinbar ist ihm die Etablierung des qualitativen Experiments als wissenschaftlich anerkanntes experimentelles Forschungsdesign nur sehr begrenzt geglückt. Sehr vereinzelt wird in Methodenlehrbüchern oder Überblickswerken auf dieses Verfahren verwiesen, doch das qualitative Experiment scheint nicht zum üblichen Kanon der

\footnotetext{
${ }^{17}$ Auszüge dieser Darstellung des qualitativen Experiments und seiner Anwendung zur Untersuchung wirtschaftswissenschaftlicher Laborexperimente wurden bereits in Haus (2018) genutzt.
} 
sozialwissenschaftlichen Methoden zu gehören (Mayring 2002; Flick et al. 1991, 1995; Lamnek 2010; Mey \& Mruck 2010; Fuchs-Heinitz et al. 1994). Dies war nicht immer so. Das qualitative und das quantitative Experiment waren zunächst verbunden: Zu Zeiten von Newton und Galilei war es auch in der naturwissenschaftlichen Forschung üblich, qualitative Formen des Experiments anzuwenden (Kleining 1986: 729). Auch in der Würzburger Schule, der Gestaltpsychologie und der Entwicklungspsychologie Jean Piagets wurde auf qualitative Experimente zurïckgegriffen (Burkhart 2010: 252).

Kleinings Überlegungen fußen auf der Annahme, dass alle sozialwissenschaftlich-empirischen Methoden, denen das Kriterium der Wissenschaftlichkeit zugeschrieben wird, auf Alltagsmethoden zurückgehen. ${ }^{18}$ Diese Alltagsmethoden werden für wissenschaftliche Untersuchungen in eine objektivere bzw. stärker intersubjektive Form überführt. Kleining verdeutlicht den Zusammenhang der Methoden in einer Pyramide (siehe Abbildung 4.1). Die Basis dieser Pyramide stellen die Alltagsmethoden dar. Durch zunehmende Abstraktion der einzelnen Situationen entwickeln sich qualitative Methoden und durch die weitere Steigerung des Abstraktionsgrades quantitative Methoden. Eine zusätzliche Ausdifferenzierung erfolgt durch die Unterscheidung zwischen aktiver und rezeptiver Auseinandersetzung mit dem interessierenden Gegenstand. Die aktiven Handlungsformen fasst Kleining unter dem Begriff Experiment, die eher passiven bzw. rezeptiven als Beobachtung. Beide sind für ihn Alltagstechniken. Sie sind „Ausdruck beider Seiten der naturwüchsigen Form der Verbindung des Menschen mit seiner Umwelt: Einerseits des Eingriffs in sie, ihrer Veränderung durch Handeln, andererseits des Aufnehmens oder Rezipierens ihrer Wirkung“ (ebd.: 726). Von den meisten Methoden gibt es sowohl qualitative als auch quantitative Verfahren. Eine Leerstelle stellt allerdings die qualitative Form des Experiments dar.

Das zentrale Definitionskriterium des Experiments ist die aktive Variation der Erhebungssituation und nicht die Suche nach kausalen Zusammenhängen. So schreibt schon Ernst Mach ([1917] 2004), den Kleining als Begründer der Methode des qualitativen Experiments ausweist: „Die Grundmethode des Experiments ist die Methode der Variation“ ([1917] 2004: 201). Kleining definiert das qualitative Experiment wie folgt: „Das qualitative Experiment ist der nach wissenschaftlichen Regeln vorgenommene Eingriff in einen (sozialen) Gegenstand

\footnotetext{
${ }^{18}$ Das Argument, dass wissenschaftliche Methoden sich nicht grundlegend von solchen des Alltags unterscheiden, wird auch in der Ethnomethodologie und in konstruktivistischen Laborstudien (Knorr-Cetina 1984) verwendet und bildet die argumentative Grundlage für das Aufbrechen der Differenz von wissenschaftlicher Rationalität und Alltagsrationalität (Siehe dazu auch Abschnitt 3.2.2).
} 


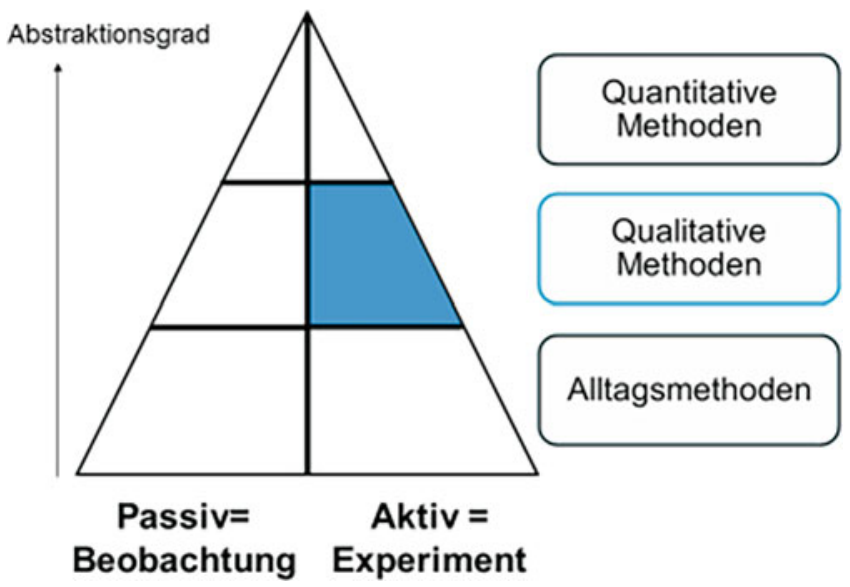

Abb.4.1 System der Methoden. (Nach Kleining 1995: 137)

zur Erforschung seiner Struktur. Es ist die explorative, heuristische Form des Experiments." (Kleining 1986: 724)

Der Eingriff in den sozialen Gegenstand ist eine notwendige Bedingung für die aktive Vorgehensweise des Experiments. Das qualitative Experiment ,geht nicht von Bekanntem, von Hypothesen aus, sondern zielt auf Neues, auf Entdeckungen. Es verwendet nicht Variablen, sondern sucht und findet Strukturen. Dies sind alle Arten von Abhängigkeiten, Beziehungen, Relationen, nicht nur kausale“ (Kleining 1986: 725). Es zielt dabei auf die Erforschung der sozialen Struktur des Forschungsgegenstands ab. Gemeint ist das für den empirischen Gegenstand Besondere, Typische und Spezifische, was ihn in seiner Eigenheit ausmacht. Kleining beschreibt dies als ,das Finden, das Aufdecken von Verhältnissen, Relationen, Beziehungen und Abhängigkeiten“ (ebd.: 725). Diese Relationen sind qualitativer Art und lassen sich meist nicht quantitativ messen, weil ,sie nicht nur Verläufe, sondern auch Negationen, Widersprüche, unstabile Abhängigkeiten, Umspringbeziehungen und Brüche einbeziehen" (ebd.). Hinsichtlich der Zielstellung des qualitativen Experiments zeigt sich somit eine deutliche Nähe zur Gattungsanalyse, die ja ebenfalls komparativ angelegt ist und nach den typischen und spezifischen Strukturen sucht, die ein empirisches Phänomen des kommunikativen Handelns auszeichnen. 
Vergleicht man das quantitative und das qualitative Experiment, werden die Differenzen zwischen beiden Methoden offensichtlich. Das quantitative Experiment geht hypothesenprüfend vor und ist auf die Aufdeckung kausaler Zusammenhänge ausgerichtet. Das qualitative Experiment hingegen geht nicht von Bekanntem und Hypothesen aus. Es ist explorativ und zielt auf die Entdeckung der Struktur des Forschungsgegenstands. Da das qualitative Experiment der Methodologie qualitativer Forschung folgt, sind Wiederholbarkeit, Vergleichbarkeit und Standardisierung keine Bedingungen für seine Durchführung. Es zielt auf die Ebene des Konkreten, Besonderen und Situativen und nicht auf die Abstraktion der einzelnen Erhebungssituationen ab. Das qualitative Experiment ist in der Methodologie der qualitativen Heuristik verankert (Kleining 1982: 227 f.). Der Forschungsprozess wird dabei als dialogisch bestimmt. Wie bei Kleinings Kategorisierung der Methoden handelt es sich auch hier um ein Zusammenspiel von passiven und aktiven Handlungsformen. Der Dialog ist gesteuert durch Fragen, die der Forschende an seinen Gegenstand richtet (aktiv) und durch die Antworten, die sich durch die Befunde des empirischen Materials ergeben. Aus den Antworten ergeben sich wieder neue Fragen, die wiederum in der Analyse des empirischen Materials zu neuen Antworten führen. Hinsichtlich des qualitativen Experiments bedeutet dies, dass die Fragen an den Forschungsgegenstand in Experimente überführt werden. Die Antworten ergeben sich wiederum durch die Befunde der Experimente und deren Analyse und bieten Anstoß für neue experimentelle Eingriffe (Fragen) (Burkart 2010: 257).

Es gibt nun verschiedene Möglichkeiten, durch einen gegenstandsadäquaten Eingriff eine Variation des Untersuchungsbereichs zu produzieren (siehe unten). Die erzeugten Variationen werden anschließend miteinander in Beziehung gesetzt, um etwas über die Struktur des Phänomens zu erfahren. Forschungsgegenstand ist somit die Gesamtheit der Veränderungen in der Struktur eines Gegenstands, die durch diese Variation erzielt werden. Stück um Stück wird in aufeinander folgenden Variationen und Vergleichen überprüft, welche Variationen zu welchen Veränderungen des Forschungsgegenstands führen. Es handelt sich um ein strikt datengeleitetes Vorgehen. Die Auseinandersetzung mit dem Forschungsgegenstand erfolgt im Sinne der Grounded Theory iterativ-zyklisch. Sie vollzieht sich in einem Wechselspiel zwischen der empirischen Datenerhebung und der Generierung neuer Forschungsfragen und -hypothesen. Durch die Offenlegung der Methoden, Intersubjektivität und Nachprüfbarkeit der Ergebnisse grenzt sich das qualitative Experiment damit deutlich von der Alltagstechnik des Experimentierens ab. 
Kleining schlägt sechs konkrete Techniken vor, die zur Erzeugung eines gegenstandsadäquaten Eingriffes genutzt werden können (Kleining 1986: 736 ff.; Kleining 1991: 265):

- Segmentation und Separation beziehen sich auf die Teilung des Gegenstandes. Der Gegenstand wird untergliedert (Segmentation) oder einzelne Bereiche werden abgetrennt (Separation).

- Bei der Kombination werden die vorgefundenen Elemente des Gegenstands auf neue Weise zusammengesetzt.

- Reduktion und Abschwächung folgen der Frage, welche Elemente eines Gegenstandes entfernt (Reduktion) oder reduziert (Abschwächung) werden können, ohne dass sich maßgeblich etwas ändert bzw. welche Elemente sensibel auf Eingriffe dieser Art reagieren und sich deutliche Konsequenzen zeigen.

- Adjektion und Intensivierung beziehen sich auf eine Veränderung des Gegenstands durch das Hinzufügen (Adjektion) oder das Verstärken (Intensivierung). Auch hier gilt es zu betrachten, ob es Teile des Gegenstands gibt, die mehr oder weniger sensibel auf Veränderungen dieser Art reagieren.

- Substitution bezieht sich auf das Auswechseln einzelner Teile des Gegenstands und das Ersetzen durch andere, um ihre strukturelle Bedeutung für den Forschungsgegenstand zu untersuchen.

- Transformation bezeichnet die Umwandlung des gesamten Gegenstands in einen anderen Gegenstand, der jedoch noch Merkmale des ersten Gegenstandes enthält. Möglichkeiten, dies umzusetzen, bestehen im Einsatz von Negationen oder Umkehrungen.

Die hier vorgestellten Techniken werden, auch von Kleining, nur als mögliche Optionen betrachtet. Es gilt, jeweils in der Auseinandersetzung mit dem spezifischen Gegenstand die geeigneten Eingriffe zu bestimmen und Stück um Stück unterschiedliche Variationen zu testen. Vor und nach dem Eingriff wird der Gegenstand deskriptiv beschrieben. Im Sinne von Kleinings Dialogprinzip stellt, wie oben ausgeführt, der experimentelle Eingriff die Frage an den Forschungsgegenstand dar. Die deskriptive Beschreibung des Gegenstands, in den eingegriffen wurde, bildet wiederum die Antwort auf den manipulativen Eingriff.

Im Rahmen meiner Forschung wurde, orientiert am Vorgehen des qualitativen Experiments, eine Serie von Experimenten konzipiert. Für den gezielten gegenstandsadäquaten Eingriff wurde dabei die Technik der Reduktion angewendet. Die Perspektive, die das qualitative Experiment auf die Geschehnisse im Labor erlaubt, kann im Rahmen der Gattungsanalyse als ein experimenteller Validitätstest der herausgestellten Rahmungsmerkmale und Verankerungsversuche betrachtet werden. Die Durchführung der Experimentreihe und die Ergebnisse werden 
aus diesem Grund im Anschluss an die wissenssoziologische Gattungsanalyse im Abschnitt 5.5 vorgestellt.

\subsection{Vorgehen der Datenerhebung}

Die unterschiedlichen Sorten von Daten, die gesammelt wurden, eint, dass sie alle gleichermaßen Versuche darstellen, relevante Dimensionen des Phänomens der kommunikativen Konstruktion des Laborexperiments analytisch greifbar zu machen.

Wie oben angeführt, war mein Vorgehen zentral durch den Anspruch geprägt, einerseits der Natürlichkeit des Phänomens Rechnung zu tragen und zugleich den Anspruch an Gegenstandsadäquanz der Forschungs- und Erhebungsmethode zu berücksichtigen. Im Fall der Datenerhebung im Labor bedingte dies eine starke Restriktion der Möglichkeiten hinsichtlich des Einsatzes bestimmter Datenerhebungsverfahren. Die Grenzen einer fruchtbaren Datenerhebung ergaben sich zum einen durch das materielle Setting des Labors und auf der anderen Seite durch zentrale Regeln bei der Durchführung von Experimenten. Zudem erschwerten paradigmatisch und methodologisch begründete Aspekte der ,Laborhygiene ' die Anwendung bestimmter technischer Hilfsmittel der Datenerhebung. Die Auseinandersetzung mit diesen Problemen führte zu einer Suchbewegung hinsichtlich eines passenden Vorgehens der Datenerhebung. Zugleich verhalfen diese Probleme jedoch dazu, über den Beitrag zu reflektieren, welchen die jeweiligen Erhebungsverfahren zur Untersuchung des Forschungsgegenstands der Rahmenbildungsprozesse im wirtschaftswissenschaftlichen Experimentallabor leisten konnten.

Von zentraler Bedeutung war für die hier vorliegende Arbeit zudem die Idee des „Theoretical Sampling“ (Glaser \& Strauss 1998: 53). Diese beschreibt im Rahmen der Grounded Theory den Prozess der Datenerhebung. Das Besondere an diesem Vorgehen besteht darin, dass nicht $\mathrm{zu}$ Beginn festgelegt wird, nach welchen Kriterien die Daten erhoben werden sollen. Es ist vielmehr so, dass ,der Forscher seine Daten parallel erhebt, kodiert und analysiert sowie darüber entscheidet, welche Daten als nächstes erhoben werden sollen" (ebd.). Die Datenerhebung erfolgt somit sukzessiv und richtet sich nach den Forschungshypothesen, die auf der Grundlage der bereits gesammelten Daten aufgestellt wurden. Um festzustellen, wie und wo mit der Datenerhebung begonnen werden kann, ist der Forschende auf eine theoretische Sensibilität gegenüber seinem Forschungsgegenstand angewiesen. Es gilt dabei, das eigene Forschungsinteresse so weit zu konkretisieren, dass auf fruchtbare Untersuchungsfälle für die Erhebung geschlossen werden kann. Zentral für das Vorgehen der Grounded Theory 
ist dabei die Methode des permanenten Vergleichs. Nachdem die ersten Daten gesammelt wurden, gilt es im weiteren Verlauf, Vergleichsfälle zu erheben. Diese dienen dazu, die aufgestellten Annahmen zu überprüfen. Durch eine Minimierung und Maximierung von Differenzen der Fälle wird dabei versucht, die relevanten Aspekte des Phänomens zu entdecken und in ihren unterschiedlichen Ausprägungen zu erfassen. Das jeweilige Forschungsinteresse bestimmt dabei maßgeblich darüber, welche Fälle als Maximal- und Minimalvergleiche herangezogen werden (Truschkat et al. 2007: 247). Der zirkuläre Prozess von Datenerhebung und -auswertung kann dann beendet werden, wenn die Forschenden den Eindruck haben, dass weitere Daten keine neuen Erkenntnisse hinsichtlich des Forschungsinteresses liefern können. Im Sinne der Grounded Theory wird dieser Aspekt als „theoretische Sättigung“ bezeichnet. Wann dieser Punkt erreicht ist, liegt dabei im Ermessen des Forschenden selbst und der ,theoretischen Sensibilität“" (Strauss \& Corbin 1996: 25) ${ }^{19}$, die er in der Auseinandersetzung mit dem Forschungsgegenstand gewonnen hat. Ganz im Sinne der iterativ-zyklischen Forschungslogik qualitativer Forschung ging mit den Prozessen der Datenerhebung dabei eine schrittweise Spezifizierung des Forschungsgegenstands selbst einher.

Im Folgenden werden die unterschiedlichen Sorten der erhobenen Daten vorgestellt und begründet, welcher Wert ihnen hinsichtlich der Untersuchung des Phänomens des ökonomischen Laborexperiments zugeschrieben wurde (Abschnitt 4.2.1). Im Anschluss daran werden die unterschiedlichen Datensorten hinsichtlich der zeitlichen Dimension ihrer Erhebung und der unterschiedlich ausgeprägten Reaktivität der Erhebungsverfahren im untersuchten Feld eingeordnet (Abschnitt 4.2.2).

\subsubsection{Unterschiedliche Sorten ethnographischer Daten}

Die nachfolgenden Ausführungen zielen darauf ab, transparent darzustellen, welche Datensorten im Forschungsprozess erhoben wurden und die Grundlage der

\footnotetext{
${ }^{19}$ Als zentrale Quellen für die theoretische Sensibilität gegenüber dem eigenen Forschungsfeld gelten persönliche und berufliche Erfahrungen, das Studium relevanter Literatur und der Prozess der Analyse selbst. Die Erhöhung der theoretischen Sensibilität gegenüber dem untersuchten Feld soll es ermöglichen, den Forschenden für die Feinheiten des empirischen Feldes zu sensibilisieren und dabei helfen zu entscheiden, welche Aspekte relevant für eine weitere Analyse sein könnten. „Erst die theoretische Sensibilität erlaubt es, eine gegenstandsverankerte Theorie zu entwickeln - und zwar schneller, als wenn diese Sensibilität fehlt" (Strauss \& Corbin 1996: 25).
} 
durchgeführten Gattungsanalyse bilden. Die nachfolgenden Unterkapitel thematisieren jeweils eine Datensorte. In den Abschnitten wird dabei expliziert, welche Verfahren der Datenerhebung genutzt wurden. Zudem wird der Umfang der jeweiligen Datenerhebung ausgewiesen sowie die Art der Operationalisierung im Sinne der Überführung in analysierbares Datenmaterial.

\subsubsection{Beobachtungsdaten durch direkte Beobachtung von Experimenten}

Diese Art von Beobachtungsdaten wurden primäre durch direkte Feldkontakte im Rahmen der Ethnographie im Labor gebildet. Die Daten basieren auf der Beobachtung von insgesamt 40 ökonomischen Laborexperimenten. Hinsichtlich der Beobachtung im Labor muss zwischen verschieden Erhebungsverfahren unterschieden werden. Denn im Verlauf des Forschungsprozesses wurden, wie oben ausgeführt, verschiedene Varianten der Beobachtung des Untersuchungsphänomens genutzt, welche zu unterschiedlichen Daten führten.

Die Operationalisierung erfolgte dabei durch das Verfassen von Feldnotizen und Memos. Hier wurden markante Geschehnisse in schriftlicher Form festgehalten und so für die nachträgliche Analyse konserviert.

Um die Natürlichkeit der Situation zu erhalten, wurden zu Beginn teilnehmende Beobachtungen verschiedener Laborexperimente durchgeführt. Der allgemeine Ablauf der Experimente, von der Ankunft der ProbandInnen vor dem Labor bis hin zum Abholen des von ihnen erspielten Gewinns, ließ sich dabei aus der Position einer teilnehmenden Beobachterin recht gut erfassen. Das räumliche Setting des Labors erschwerte jedoch die Beobachtung während des Experiments. Aufgrund der hohen Trennwände war eine detaillierte Beobachtung einzelner ProbandInnen nur möglich, wenn man sich genau hinter ihrem Arbeitsplatz befand. Meine dauerhafte Positionierung an dieser Stelle führte bei den so Beobachteten zu erheblicher Reaktanz. Die ProbandInnen verharrten, drehten sich mehrfach zu mir um und zeigten sichtliche Irritation. Die auf diese Weise erhobenen Daten ermöglichten es daher nicht, genauer zu betrachten, welche Handlungen die ProbandInnen im Detail vollzogen. Es wurde zwar ersichtlich, dass die Abläufe im Labor einer gewissen Ordnung folgten, die Feinheiten aber blieben verborgen.

Diese Art der Datenerhebung stellte einen ersten Schritt in der Auseinandersetzung mit dem Feld dar. Der oben dargestellte Wechsel meiner Feldrolle ermöglichte es, in einer späteren Phase Beobachtungen aus der Perspektive einer Laborforscherin zu vollziehen. Gerade die Phase meiner Schulung war besonders erkenntnisreich. Hier waren jedoch weniger die allgemeinen Abläufe von Interesse, sondern die Anforderungen, die sich im Umgang mit den ProbandInnen ergaben. Diese Beobachterposition lieferte entscheidende Erkenntnisse bezüglich 
der Frage, wie und mittels welcher Methoden die Situation des Experiments kontrolliert und aufrechterhalten wurde und wie krisenhafte Situationen entstanden und situativ gelöst wurden. Zugleich wurde durch den intensiveren Kontakt mit den ProbandInnen ersichtlich, dass auch sie deutliche Normalitätserwartungen bezüglich der Geschehnisse im Labor hatten.

Die anfänglichen Beobachtungen im Labor ermöglichten es, zeitgleich zum Ablauf des Experiments Feldnotizen und Beobachtungsprotokolle anzufertigen, in denen auffällige Aspekte der Laborsituation vermerkt wurden. In unterschiedlichen Experimenten wurden dabei verschiedene Gesichtspunkte intensiver thematisiert, beispielsweise die Phase der Ankunft der ProbandInnen und ihrer Randomisierung, ihrer Orientierung im Labor selbst oder das allgemeine Ablaufschema der Experimente. Weitere Feldnotizen widmeten sich den Vorgängen, die von Seiten der ExperimentatorInnen im Rahmen der Vorbereitung der Experimente relevant waren.

Durch meine stärkere Involvierung in die Durchführung der Datenerhebung im Labor war es als teilnehmende Beobachterin nur noch begrenzt möglich, Feldnotizen zeitgleich mit dem Experiment selbst zu verfassen. Die Feldnotizen konnten aus diesem Grund erst nachträglich angefertigt werden. Einige Notizen dieser Erhebungsphase fokussieren einzelne (häufig krisenhafte) Episoden des Laborgeschehens und die situativen Lösungen durch die Handelnden. Andere Textdokumente sind stark durch eine autoethnographische Perspektive geprägt, die der eigenen Durchführung der Experimente entspringt und thematisiert Fragen und Erkenntnisse, die sich durch die Arbeit im Labor ergaben. Hier handelt es sich stärker um Memos, die einzelne Aspekte systematisch erfassen und verschiedene empirische Einzelsituationen überspannen.

\subsubsection{Beobachtungsdaten durch Videoaufnahmen}

Als alternative Möglichkeit Beobachtungsdaten zu erheben, wurde der Einsatz von Videokameras genutzt. Zu diesem Zweck wurde an der Decke des Labors eine Kamera installiert. Diese Deckenkamera erlaubte es, die Abläufe im Labor aus einer Vogelperspektive aufzuzeichnen. Zusätzlich wurden an vier Arbeitsplätzen am oberen Rand der Bildschirme Webcams installiert, um die Handlungen der ProbandInnen genauer zu erfassen.

Hinsichtlich der Erhebung von Videodaten fanden zwei Erhebungsphasen statt. Der erste Teil verfolgte dabei das Ziel, auszuprobieren, wie Videos im Experimentallabor erhoben werden und welchen Wert diese Daten für eine qualitative Analyse haben könnten. Es wurde dafür eine Versuchsanordnung ausgewählt, deren Durchführung ich im Rahmen meiner ethnographischen Arbeit im Labor selbst bereits mehrere Male beobachtet hatte. Es wurden nacheinander drei Sessions erhoben, in denen zusätzlich mittels einer speziellen Software die Abläufe auf 
dem Computerbildschirm aufgezeichnet wurden. ${ }^{20}$ Bei der Erhebung der Videodaten kam es jedoch zu verschiedenen lehrreichen Problemen. Zum einen zeigte sich, dass die Ausrichtung der Webcams bei einem Teil der Aufnahmen nicht der Positionierung der Handelnden vor dem Bildschirm entsprach. Die gefilmten Ausschnitte bildeten aus diesem Grund in einigen Fällen nur Teile der Gesichter und Köpfe der ProbandInnen an ihren Arbeitsplätzen ab und waren für die weitere Analyse kaum verwertbar. Auch war die Auswahl der Arbeitsplätze, an denen die Webcams installiert wurden, suboptimal. ${ }^{21}$ Neben dem allgemeinen Ablauf, der sich durch die Deckenkamera gut erfassen ließ, war diese zugleich in der Lage, die Abläufe an einigen Arbeitsplätzen in der Mitte des Labors sehr detailliert zu erfassen. Die für die Webcam ausgewählten Arbeitsplätze lagen jedoch am oberen Rand des Labors. Zugleich bedingte die mangelnde Softwareschulung eines Laborkollegen, dass nicht alle Aufnahmen in der zweiten Session rechtzeitig gestartet wurden. Trotz dieser Probleme lieferte bereits diese erste Erhebung im Labor wichtige und detailreiche Daten für die Analyse. Es wurden durch die Deckenkamera 350 Minuten Videomaterial im Labor aufgezeichnet. Die für die Videoanalyse, verwertbaren' Aufnahmen der Webcams umfassten 630 Minuten.

Die zweite Erhebung erfolgte im Rahmen des qualitativen Experiments. Hier wurden aus den Fehlern gelernt und die Erhebung entsprechend angepasst. Die Webcams wurden an Arbeitsplätzen in der Mitte des Labors installiert. Es wurden dabei nacheinander vier Sessions gefilmt und zugleich an jeweils vier Arbeitsplätzen Aufnahmen mittels Webcams gemacht. Insgesamt wurden in diesen vier Sitzungen 160 Minuten Videomaterial durch die Deckenkamera und entsprechend 620 Minuten durch die Webcams aufgezeichnet.

Die so generierten Daten bieten einen entscheidenden Vorteil gegenüber den Feldnotizen und Protokollen, die üblicherweise im Rahmen der teilnehmenden Beobachtung angefertigt werden, da sie den Forschenden in Zuge der Datenerhebung weit weniger zu einer Reduktion und Auswahl der relevanten Aspekte nötigen. Sicher muss auch bei einer Erhebung von Videodaten geklärt werden, wer, wo und wann gefilmt werden soll. Doch ist diese Auswahl getroffen, ist durch das ,Einfrieren` des natürlichen Handlungsvollzugs die Möglichkeit seiner

\footnotetext{
${ }^{20}$ Es handelte sich dabei um eine Screen Capture Software namens „Snagit“.

${ }^{21}$ Der Grund für die Auswahl lag darin, dass sich diese Arbeitsplätze in Beobachtungsnähe des Platzes befanden, an dem sich die ExperimentatorInnen während des Experiments aufhalten. $\mathrm{Zu}$ diesem Zeitpunkt nahm ich an, dass die Videobeobachtungen so durch teilnehmende Beobachtungen aus einer anderen Perspektive ergänzt werden könnten. Im Vergleich der Optionen zeigte sich jedoch der qualitative Mehrwert einer Datentriangulation der detaillierten Videoaufzeichnung von Deckenkamera und Webcam.
} 
Konservierung und Reproduktion deutlich erhöht. ${ }^{22}$ Mittels der Videodaten war es möglich, die Abläufe im Labor für eine spätere Analyse zu konservieren und so in ihrem sequentiellen Vollzug immer wieder zu betrachten. Gerade in einer Phase der Forschung, in der sich der Forschungsgegenstand selbst noch nicht klar herauskristallisiert hat und noch unsicher ist, welche Aspekte für eine spätere Analyse von besonderer Relevanz sind, war dies von enormem Wert. Zugleich ermöglichte es die Aufzeichnung von Videodaten, diese in gemeinsamen Datensitzungen mit anderen Forschenden zu betrachten. Dies bereicherte sowohl den eigenen Interpretations- und Erkenntnisprozess als auch die Validität der eigenen Deutung und Analyse enorm (siehe dazu auch Wilke \& Lettkemann 2016).

\subsubsection{Beobachtungsdaten durch direkte Beobachtung der experimentellen WirtschaftsforscherInnen}

Weitere ethnographische Daten wurden durch die Beobachtung und Interaktion mit den wirtschaftswissenschaftlichen ForscherInnen und Labormitgliedern generiert. Das Brückenprojekt, in welches ich eingebunden war, ermöglichte es, dass ich in Phasen der Konzeption und Vorbereitung von verschiedenen Serien von Experimenten eingebunden war. In den drei durchgeführten Serien von Experimenten wurden dabei in gemeinsamen Projektsitzungen die unterschiedlichen Phasen der Themenauswahl, Konzeption, Durchführung und Auswertung der Experimente gemeinsam erörtert. Im Verlauf der Projektarbeit bezog ich zudem für zehn Monate einen Büroarbeitsplatz in der Abteilung der wirtschaftswissenschaftlichen ForscherInnen und nahm am Abteilungsalltag teil. Ich teilte mir dabei das Büro während dieser Zeit mit unterschiedlichen Forschenden und diskutierte mit ihnen darüber, welche Experimente sie planten oder gerade durchführten.

Es wurden in dieser Zeit vielfältige Feldnotizen und Memos angefertigt. Diese thematisieren vorrangig Aspekte, die sich in der Zusammenarbeit mit den ökonomischen ForscherInnen als relevant herausstellten. Es handelt sich dabei häufig um konzeptionelle Annahmen und ihre Überführung und Umsetzung im Rahmen der Experimente selbst. Weitere Aspekte sind Probleme und Irritationen, die meine eigene Datenerhebung im von mir beforschten Feld provozierte. Diese Notizen verweisen zugleich auf grundlegende Differenzen der Forschungsperspektiven der Projektpartner und methodologisch begründete paradigmatische Beschränkungen im Vorgehen der Laborexperimente. Ein Beispiel dafür ist der Fall der Erhebung von Videodaten im Labor: Vielfältige Aushandlungen und

\footnotetext{
${ }^{22}$ Auf diesen Aspekt wurde bereits im Abschnitt 4.1.2 im Zusammenhang mit der Videographie eingegangen.
} 
Gespräche mit unterschiedlichen Personen waren hier im Vorlauf der Datenerhebung notwendig. Der Prozess zog sich über sechs Monate hin, war jedoch selbst sehr erkenntnisreich.

Eine weitere Datenquelle, welche aus diesen Situationen hervorging, waren Audiomittschnitte aus gemeinsamen Projektsitzungen. Mit Hilfe eines Diktiergerätes wurden mehrere gemeinsame Projektsitzungen und Gespräche mit den ökonomischen KollegInnen aufgezeichnet. Diese Gespräche thematisieren die Auswahl, Konzeption, Durchführung und Auswertungen von Experimenten, die im Verlauf des Experiments durchgeführt wurden, sowie Präsentationen und Berichte des Projekts, die für verschiedene Anlässe ausgearbeitet werden mussten. Es liegen hier insgesamt 20 Stunden Audiomaterial vor. Aufgrund des großen Datenvolumens wurden diese Daten nur selektiv und ausschnittweise transkribiert, soweit sie für die Analyse relevante Aspekte thematisierten. Die Daten liegen aber vollständig in digitaler Form vor.

Aus diesen Daten konnten entscheidende Erkenntnisse darüber gewonnen werden, welche Aspekte für die Konzeption von Experimenten relevant sind und wie die häufig paradigmatisch begründeten Ansprüche der Forschenden in der Praxis des Labors umgesetzt werden. Diese Daten lieferten somit reichhaltige Hintergrundinformationen und führten zu einer entscheidenden Vertiefung meines ethnographischen Wissens hinsichtlich der Perspektive der wirtschaftswissenschaftlichen ForscherInnen auf das Geschehen im Labor.

\subsubsection{Interviews mit ProbandInnen}

Aufgrund des Mangels an beobachtbarer Kommunikation und Interaktion im Labor konnte aus den Beobachtungsdaten nicht darauf geschlossen werden, welche praktischen Methoden die ProbandInnen bei ihrer Entscheidungsfindung nutzten und wie sie die Situation des Laborexperiments in ihrer Typizität wahrnahmen. Das für die ProbandInnen geltende Kommunikationsverbot im Labor ${ }^{23}$ machte es zudem unmöglich, diese ihre Handlungen simultan zu ihrem Vollzug kommentieren zu lassen. Aus diesem Grund wurden Interviews mit den ProbandInnen geführt - so sollten mehr Informationen über die Entscheidungshintergründe der ProbandInnen erhoben und einige aus den Beobachtungen

\footnotetext{
${ }^{23}$ Der hier verwendete Begriff des „Kommunikationsverbotes“ ist als Feldbegriff der Forschenden zu verstehen. Er besitzen hier eine Bedeutung und Verwendung, die sich von der theoretischen und konzeptionellen Position unterscheidet, die in dieser Arbeit verwendet wird. Kommunikation wird in dieser Verwendung (stark) auf Sprache und verbale Entäußerungen reduziert. Aus Sicht der Forschenden meint Kommunikationsverbot hier spezifischer, dass die ProbandInnen nicht miteinander sprechen dürfen und ihre Handlungen und Ergebnisse nicht verbal kommentieren sollen.
} 
gewonnene Hypothesen im Dialog mit ihnen überprüft werden. ProbandInnen beschrieben hier ihre Vorerfahrungen mit Experimenten, ihre Wahrnehmung von Laborregeln sowie Wissenshintergründe und Strategien, die sie im Rahmen von Experimenten anwendeten. ${ }^{24}$

Nur die ersten sechs offenen Interviews wurde vor der Durchführung eines Experiments geführt. Alle weiteren Interviews fanden direkt im Anschluss an ein spezifisches Experiment statt. Die gemeinsame Erfahrung der Experimentsituation stellte einen zentralen Gesprächshintergrund dar, auf welchen im Interview Bezug genommen wurde. Aus diesem Grund war es möglich, über Entscheidungspraktiken und die Wahrnehmung ganz konkreter Entscheidungssituationen zu sprechen. Diese Thematisierung experimentspezifischer Aspekte mit den ProbandInnen verwies deutlich auf eine Differenz zwischen den Annahmen und Ansprüchen, die die ökonomischen ForscherInnen der Konzeption der Experimente zugrunde legten, und der Wahrnehmung und dem Verständnis der teilnehmenden ProbandInnen eben dieser Aspekte.

Im Verlauf des Forschungsprozesses wurden verschiedene Interviewleitfäden ausgearbeitet und genutzt. In 62 Interviews wurden dabei Interviewdaten durch Gespräche mit 146 ProbandInnen erhoben. Hinsichtlich des Samplings muss darauf verwiesen werden, dass die Teilnahme an den Interviews den ProbandInnen freigestellt war und insofern eine gewisse Selbstselektion nicht ausgeschlossen werden kann. Am Ende des eigentlichen Experiments im Labor, parallel zum Ausfüllen ihrer Quittungen, wurden die ProbandInnen darüber informiert, dass einige von ihnen im Anschluss die Möglichkeit hätten, an einem Interview teilzunehmen und dass sie dafür zusätzlich mit 5 Euro entlohnt werden würden. Ich stellte im Folgenden im Labor selbst die Interviewgruppen zusammen. Dies erfolgte, indem ich gezielt ProbandInnen ansprach. Die Interviewsituation selbst wurde dabei verschiedentlich variiert, um die Gefahr von Intervieweffekten zu reduzieren. So wurden beispielsweise verschiedene Personen für die Durchführung der Interviews eingesetzt oder die Anzahl der in einem Interview gleichzeitig interviewten ProbandInnen variiert. ${ }^{25}$

\footnotetext{
${ }^{24}$ Unter Berücksichtigung des Datenschutzes der Interviewten und zur Anonymisierung des Datenmaterials wurden die ProbandInnen stets zu Beginn der Interviews gebeten, sich selbst ein Alias zu geben. Mit diesem wurden sie im Verlauf des Interviews angesprochen. Ob es sich dabei um ihren tatsächlichen Namen handelte, wurde nicht erfragt. Die von den Interviewten verwenden Alias wurden auch in den Interviewausschnitten genutzt, die im Analyseteil zur Darstellung eingesetzt werden.

${ }^{25}$ Es wurden dabei jeweils zwischen 1 und 6 ProbandInnen zugleich interviewt Die Entscheidung für ein Gruppeninterview mit einer höheren Anzahl an Teilnehmenden hatte auch forschungspraktische Vorteile. Gerade in Erhebungssituationen, in denen ich keine weiteren
} 
Gerade zu Beginn bemühte ich mich um eine Gendergleichverteilung. Der Überblick über die Datenbasis offenbart jedoch, dass insgesamt 80 Männer und nur 65 Frauen interviewt wurden. Dieser Bias resultiert daher, dass im Verlauf der Datenerhebung andere Charakteristika stärker die Auswahl der Interviewpartner lenkten. ${ }^{26}$ In einigen Experimenten wurden gezielt Interviewpartner eingeladen, die im Rahmen des Experiments bestimmte Rollen eingenommen hatten (Mindestlohn, Exklusivitätsexperiment), oder solche, die im Rahmen des Experiments ein Spielerpaar gebildet hatten. Im Zuge der Auswahl der Interviewpartner für die Erhebung zum qualitativen Experiment wurden gezielt die ProbandInnen interviewt, die auch an den per Webcam gefilmten Arbeitsplätzen saßen. Dies folgte dem Ziel, einen möglichst dichten Datensatz zu generieren.

Im Folgenden wird kurz auf die Entwicklung und Weiterentwicklung der Interviewleitfäden im Rahmen der einzelnen Erhebungsreihen von Interviews eingegangen. Unter Berücksichtigung der erhobenen Daten und im Wechselspiel mit dem eigenen Erkenntnisfortschritt wurde der Leitfaden dabei schrittweise abgewandelt, um der Fokussierung der Forschungsfrage und des Forschungsziels Rechnung zu tragen. An den Leitfäden und ihrer Entwicklung zeigt sich dabei deutlich, dass der Forschungsprozess von einem iterativ-zyklische Vorgehen im Sinne der Grounded Theory geprägt war. ${ }^{27}$

\section{Erste offene Interviews}

Anfangs handelte es sich um kurze und offene Interviews von circa $10 \mathrm{Minu}-$ ten Länge. Es wurden vorbereitend nur grobe, inhaltliche Themenkomplexe festgelegt. Diese Interviews thematisierten die allgemeinen Erfahrungen der Interviewten mit Experimenten und der Übertragbarkeit von Entscheidungen im Labor und auf den Alltag.

Interviewer zu Verfügung hatte, war es mir pro Session ja nur möglich, ein Interview zu führen. Eine Erhöhung der Gruppengröße bedingte dabei, dass ich mehr Teilnehmende befragen konnte. Entsprechend waren die Gruppeninterviews im Vergleich zu den Interviews in Zweiergruppen jedoch auch deutlich länger.

${ }^{26}$ Im Allgemeinen ist die Geschlechterverteilung der Teilnehmenden im Labor jedoch sehr ausgeglichen. Da die Zuweisung zu den einzelnen Arbeitsplätzen und Rollen jedoch randomisiert ist und ich keine Vollerhebung der Experimentteilnehmenden getätigt habe, ist diese Verzerrung entstanden.

${ }^{27}$ Die Erhebung verteilte sich, wie folgt, auf die einzelnen Experimentserien: offene Interviews und Beobachtungen verschiedener Experimente $(n=6)$, Endowment-Effekt ,Tassen und Pralinen“ $(\mathrm{n}=12)$, Endowment-Effekt „Exklusivitätsexperimente“ $(\mathrm{n}=41)$, Mindestlohn ( $\mathrm{n}$ $=18)$, Endowment-Effekt ,USB-Stick“ $(\mathrm{n}=46)$, qualitatives Experiment $(\mathrm{n}=24)$. 


\section{Interviews nach dem Pilotexperiment „Tassen und Pralinen“}

Im Rahmen des Brückenprojekts wurden zwei Sessions als Pilotexperiment der Experimentserie „Tassen und Pralinen“ zum Thema Endowment- Effekt durchgeführt. Im Anschluss an diese Experimente fanden jeweils Gruppeninterviews mit sechs Teilnehmenden statt. Inhaltlich wurden hier die allgemeinen Fragen wieder aufgegriffen. Zusätzlich wurden die ProbandInnen gebeten, mitzuteilen, wie und auf Grundlage welcher Überlegungen sie sich für oder gegen den Tausch eines bestimmten Objekts entschieden haben.

\section{Interviews in der Experimentserie „Exklusivitätsexperiment““}

Im weiteren Verlauf des Projekts wurde für die nächste Erhebungsphase ein Leitfaden (LF1) zusammengestellt, auf dessen Basis halb-strukturierte Interviews geführt wurden. Dieser Leitfaden wurde begleitend zur Experimentserie „Exklusivitätsexperimente“ eingesetzt. Auch dieser Interviewleitfaden war explorativ ausgerichtet und zielte auf die Erhebung relevanter Details des Laborgeschehens ab. Zentrale Themenkomplexe dieser Interviews bildeten Ablauf und Regeln im Laborexperiment und die Wissensbasis, welche ProbandInnen zur Bewältigung der gestellten Entscheidungssituationen heranzogen. Zudem wurden erste Hypothesen hinsichtlich potenzieller Rahmungsfaktoren getestet. So wurde in den Interviews thematisiert, ob aus Sicht der ProbandInnen ein Zusammenhang zwischen Spielen und ihrem Handeln und Entscheiden im Rahmen des Laborexperiments ein Zusammenhang bestand. Da im Rahmen der Exklusivitätsexperimente auch ein Fragebogen eingesetzt wurde, welcher Fragen zum Thema Spiele stellte, konnte auch in den Interviews direkt auf diesen Aspekt Bezug genommen werden.

\section{Interviews nach den „Mindestlohn-Experimenten“}

Im Anschluss an die erste Erhebung von Videodaten wurden Interviews mit einem weiterentwickelten Leitfaden LF3 durchgeführt. Direkt im Anschluss an das Experiment wurden jeweils in Zweiergruppen Interviews mit Teilnehmenden geführt. Ich wurde dabei durch zwei weitere InterviewerInnen unterstützt, sodass in den drei Sessions insgesamt 18 ProbandInnen interviewt werden konnten. Der Leitfaden LF3 entsprach zu großen Teilen der Variante LF2, die auch bei den „Exklusivitätsexperimenten“ genutzt wurde. Zusätzliche Elemente bildeten Fragen zur Thematik des monetären Anreizes, der Rollen und Rollendistanz im Labor sowie Nachfragen zur Reaktanz durch den Einsatz von Videokameras. Reduziert wurden die Fragen im allgemeinen Bereich und bezüglich des potenziellen Zusammenhangs von Spielen und Experimenten. 


\section{Interviews nach der Experimentserie ,Endowment-Effekt USB-Stick“}

Die dritte Serie von Experimenten im Rahmen des Projekts befasste sich ebenfalls mit dem Endowment-Effekt. Der Interviewleitfaden LF3a basierte grundlegend auf seinen Vorgängern aus den anderen Erhebungsphasen. Der Fragenblock zum Zusammenhang zwischen Spielen und dem Experiment wurde allerdings aufgrund der Ergebnisse der vorherigen Interviews weggelassen. Der Fragenblock zum Einfluss des monetären Anreizes wurde an das Ende des Interviews verschoben und um eine Frage erweitert. ${ }^{28}$ Die spezifischen Fragen zum Experiment wurden der neuen Serie angepasst und thematisierten den Weg der Entscheidungsfindung für den Kauf- und Verkaufspreis von Lotterielosen und dem USB-Stick. Der Ausbau dieses Fragenbereichs verfolgte das Ziel, die experimentspezifischen Entscheidungssituationen und -hintergründe der ProbandInnen detaillierter $\mathrm{zu}$ erheben. Nach den ersten 20 Interviews wurde der Leitfaden LF3a wiederum etwas verändert. Diese abgeänderte Form wurde als LF3b bezeichnet. Der Anfangsteil allgemeiner Fragen zum Laborexperiment wurde hier deutlich reduziert. Grund dafür war, dass sich hier eine deutliche theoretische Sättigung bezüglich der Antworten zeigte. Eine zweite Variante des Leitfadens (LF3b) wurde dabei ausschließlich für Sessions verwendet, in denen im Verlauf der Experimente in Zweiergruppen face-to-face das Spiel Vier-Gewinnt gespielt wurde. Aus diesem Grund wurden spezifische Fragen dazu gestellt, inwiefern dieses Spiel das Entscheidungsverhalten und die Situation des Experiments beeinflusste. Ein zentraler Fokus lag des Weiteren auf den Fragenbereichen zum Thema Rollen im Labor und dem monetären Anreiz.

\section{Interviews der Experimentserie ,Qualitatives Experiment““}

Die einzelnen Sessions entsprachen inhaltlich dem Baseline Treatment der Experimentserie „Endowment-Effekt USB-Stick“. Es handelte sich somit um eine Variante des Experiments ohne das zusätzliche Element eines Spiels, sodass auf diese Fragen in den Interviews verzichtet wurde. Aus diesem Grund wurde für die Erhebung eine Abwandlung des Leitfadens LF3a genutzt. Da zugleich Videoaufzeichnungen der Abläufe im Labor und von einzelnen Arbeitsplätzen erhoben

\footnotetext{
${ }^{28}$ Dies geschah, um zu überprüfen, ob eine frühzeitige Fokussierung dieses Aspekts zu einem Interview-Effekt führen würde Als Interview-Effekt wird eine Verzerrung der Ergebnisse bezeichnet, welche durch die Interviewsituation selbst produziert ist. In diesem Fall sollte gesichert werden, dass vom Themenbereich des monetären Anreizes kein Ausstrahlungs- oder Positionseffekt (Kromrey2009: $361 \mathrm{f}$.) ausgeht und die thematische Fokussierung so bedingt, dass auch alle nachfolgenden Fragen primär unter dem Gesichtspunkt des monetären Anreizes thematisiert werden.
} 
wurden, beinhaltete der Leitfaden Nachfragen zur Reaktanz durch die Videoaufzeichnung. Des Weiteren wurde eine Frage zur Verständlichkeit der Instruktionen und der einleitenden Erklärungen aufgenommen. Diese Leitfadenvariante wurde als LF3c bezeichnet.

\subsubsection{Standardisierte Fragebögen}

Am Ende eines Experiments wird üblicherweise ein kurzer Fragebogen von den ProbandInnen ausgefüllt, welcher sozio-demographische Daten wie Alter, Geschlecht, Studienfach, Geschwisteranzahl erfasst und ein oder zwei Freifelder für Bemerkungen zum Experiment enthält. Dieser Fragebogen wurde im Rahmen von zwei der drei Experimentserien stark ausgeweitet. Es handelte sich dabei um Fragen, die zusätzliche Informationen zu den im Experiment untersuchten Hypothesen liefern sollten. Im ersten Fall wurden primär geschlossene Fragen verwendet, im zweiten Fall nahezu ausschließlich offene Fragen. Gerade der zweite Fragebogen, der sich mit spezifischen Fragen zu den Entscheidungssituationen im Experiment befasste, thematisierte dabei Aspekte, die auch im Rahmen der Interviews besprochen wurden. Die Ergebnisse dieser Fragebögen ermöglichten es, anhand einiger Aspekten zu überprüfen, ob Erkenntnisse der Interviews auch auf die gesamte Gruppe der Teilnehmenden übertragbar waren. Sie dienten somit der Überprüfung bzw. Validitätssteigerung der Ergebnisse der Interviews.

Zudem bildeten die Fragebögen und ihre Ergebnisse in den gemeinsamen Projektsitzungen einen wichtigen und erkenntnisreichen Bezugspunkt. Dies gilt insbesondere für die Ergebnisse des Fragebogens der Experimentserie „EndowmentEffekt-USB-Stick“. ${ }^{29}$ Die qualitative soziologische und die quantitative wirtschaftswissenschaftliche Bezugnahme und Interpretation der Ergebnisse dieser Fragebögen verlief hier sehr unterschiedlich. Diese Kontroverse führte jedoch zu

\footnotetext{
${ }^{29}$ Das Experiment zielte darauf ab, die ProbandInnen dazu zu bewegen, genau den Preis für ein angebotenes Objekt anzugeben, welcher ihrer persönlichen Bewertung entsprach. Dieser beinhaltete 9 bzw. 10 Fragen zum Vorgehen der ProbandInnen bei der Bewältigung der Entscheidungssituationen, mit denen sie sich im Experiment auseinandersetzen mussten. In 8 von 19 Sessions $(n=182)$ wurde eine zehnte Frage gestellt: „Wenn Sie sich hätten entscheiden können, ob Sie den USB-Stick oder Geld (in der Höhe Ihres Gebotes) behalten möchten, was hätten Sie bevorzugt?" Diese Frage war der zentrale Gegenstand der nachfolgenden Diskussionen der Projektgruppe. Hier sollte überprüft werden, ob die ProbandInnen den im Labor eingesetzten BDM-Mechanismus richtig verstanden und angewendet hatten. Nur $15 \%$ der Befragten gaben hier jedoch die Antwort, die auf eine Übereinstimmung mit dem BDM-Mechanismus hinwies. Für eine erste Beschreibung der Experimentsserie „Endowment-Effekt-USB-Stick“ siehe 4.1.1.
} 
einer deutlichen Explikation paradigmatischer Grundannahmen der wirtschaftswissenschaftlichen ForscherInnen hinsichtlich des Handelns der ProbandInnen und des im Labor genutzten rationalen Akteursmodells.

\subsubsection{Prozessproduzierte Dokumente}

Als prozessproduziert gelten solche Daten, die ohne das Zutun des Forschenden entstanden sind. Diese Datensorte umfasst in meinem Fall schriftliche Dokumente, die im Rahmen der Experimente und ihrer Vorbereitung produziert wurden. Besonders die schriftlichen Instruktionen, die in den beobachteten Experimenten verwendet wurden, sind für die Untersuchung relevant. Weitere Datenquellen bilden Screenshots der Experimente, Werbe- und Informationsmaterialien für die Akquise und Rekrutierung von ProbandInnen und ein Dokument mit Richtlinien zu Durchführung von Experimenten in diesem Labor. Diese Dokumente erlauben es, die Selbstbeschreibung und die typische sprachliche Praxis der experimentellen WirtschaftsforscherInnen zu erfassen und zugleich detailliert zu analysieren.

Hinsichtlich der ethnographischen Untersuchung des Laborgeschehens werden auch die im Zuge der Experimente produzierten quantitativen Daten als prozessproduzierte Daten betrachtet. Diese geben Auskunft über die Zeitdauer einzelner Entscheidungsfindungen von ProbandInnen. Zugleich halten sie fest, welche Auswahl die Teilnehmenden in bestimmten Situationen getroffen haben oder welche Werte sie eingegeben haben.

Für meine eigene Untersuchung waren diese quantitativen Daten nur insofern von Belang, als dass sie in Kombination mit anderen Daten bestimmte Fragen aufwarfen oder Anstöße für vielfältige Diskussionen in der Projektgruppe lieferten. Der Erkenntnisgewinn durch diese Daten bestand vorrangig darin, zu erfahren, welche Deutung die ökonomischen ForscherInnen diesen beimessen. Diese Deutungen zeigten sich vielfach darin, wie sie in den gemeinsamen Projektsitzungen argumentativ auf die Daten Bezug nahmen. Eine spezifische Auswertung dieser Daten und ein Vergleich zwischen den unterschiedlichen Treatmentvariationen war somit zwar Gegenstand der Diskussion im Projekt, wurde im Rahmen der hier vorliegenden Analyse jedoch nicht vorgenommen. Der Grund besteht darin, dass die vorliegende Arbeit den Rahmenbildungsprozess im Labor zum Brennpunkt der Analyse macht. 


\subsubsection{Differenzen der Datensorten}

Die verschiedenen Datensorten, die zur Analyse herangezogen werden, eint, dass sie alle die ethnographische Wissensbasis vergrößern und auf diese Weise die Analyse bereichern. Sie differieren jedoch dabei in unterschiedlichen Dimensionen. Bestimmte Datensorten wurden beispielsweise im Labor selbst erhoben, andere jedoch außerhalb des eigentlichen Experiments und zeitlich versetzt. Eine weitere zentrale Dimension ist die unterschiedliche Stärke der Reaktivität der Verfahren der Datenerhebung.

\subsubsection{Diachrone oder synchrone Betrachtung der Prozesse}

Während einige Erhebungsmethoden direkt im Labor angewendet wurden und die Geschehnisse in ihrem natürlichen Vollzug erfassten, thematisieren andere Daten zwar die Geschehnisse im Labor, wurden jedoch zeitlich versetzt erhoben. Die Einordnung der erhobenen Datensorten als synchrone oder diachrone Daten zielt auf die Explikation eben dieses zeitlichen Verhältnisses zur Laborsituation ab und wird in Abbildung 4.2 veranschaulicht.

Die direkten Beobachtungsdaten aus dem Labor, die von mir selbst erhoben wurden und das Zusammenspiel von ProbandInnen und Labormitgliedern erfassten, stellen dabei synchrone Daten dar. Diese geben die natürlichen Abläufe des Experiments wieder. Den Videodaten kommt dabei eine herausgehobene Stellung zu, da sie im Unterschied zu Feldnotizen und Memos von teilnehmenden Beobachtungssituationen - nicht bereits im Erhebungsprozess durch eine Verschriftlichung zur Komplexitätsreduktion zwangen. ${ }^{30}$

Die Interviews wurden zwar bereits kurz nach den Experimenten durchgeführt, die Berichte, die die Interviewten dort über ihre Entscheidungsprozesse abgaben, sind jedoch nicht dem Entscheidungsprozess selbst gleichzusetzen, der im Labor stattfand. Es sind - im Schützschen Sinne - Erinnerungen und Rekonstruktionen der eigenen Handlungen, die im Labor abliefen und damit zugleich Interpretationen, in welchen die ProbandInnen ihr Handeln (möglicherweise zu stark) rationalisierten.

Die gemeinsamen Projektsitzungen mit den experimentellen WirtschaftsforscherInnen brachten ebenfalls Beobachtungsdaten hervor, fanden jedoch zeitlich verschoben zu den Experimenten statt. Auch wenn episodenhaft einzelne Vorfälle der Experimente thematisiert wurden, verliefen die Diskussionen üblicherweise auf einem höheren Abstraktionsniveau bezüglich der Vorkommnisse im Labor. Thematisiert wurden hier stärker konzeptionelle Annahmen und praktische Wege,

\footnotetext{
${ }^{30}$ Auf diesen Aspekt wurde bereits ausführlicher im Abschnitt 4.1.2 eingegangen.
} 
um bestimmte Aspekte im Labor umzusetzen. Bei der Diskussion von Ergebnissen wurden nicht einzelne Erhebungen im Labor fokussiert, sondern die Analyse und der Vergleich ganzer Versuchsanordnungen.

$\mathrm{Zu}$ guter Letzt bildeten quantitative Daten und Dokumente bestimmte Aspekte des Untersuchungsphänomens ab. In zeitlicher Hinsicht scheint die Frage, wann ich diese Daten erhoben habe, keinen Einfluss auf den Wert dieser Daten zu haben. Es ist jedoch durchaus wichtig zu wissen, wie und wann diese Dokumente im Prozess des Experiments selbst erhoben oder eingesetzt wurden. So werden die Fragebögen beispielsweise kurz vor dem Ende des datenerhebungsrelevanten Abschnitts des Experiments ausgefüllt und somit kurz bevor den Teilnehmenden ihr Spielgewinn ausgezahlt wird.

synchron

\section{Projektsitzungen}

Interview

primäre Beobachtungen

im Labor

Videoaufnahmen
Unbeeinflusst vom

Erhebungszeit-

punkt: Quantitative

Daten, Dokumente

und Fragebögen

diachron

Abb.4.2 Zeitliches Verhältnis von Erhebung der Datensorten und Experiment

\subsubsection{Reaktivität und Reaktanzwahrnehmung der eingesetzten Methoden}

Reaktivität bezeichnet den Einfluss des Datenerhebungsvorgangs auf die durch ihn produzierten Daten (Kromrey 2009: 503). Als nicht-reaktive Verfahren werden dabei solche bezeichnet, bei denen der Prozess ihrer Erhebung keinen Einfluss auf das untersuchte Phänomen hat (ebd.). Reaktive Verfahren der Datenerhebung 
sind hingegen solche, die durch den Prozess der Datenerhebung die resultierenden Daten selbst beeinflussen (ebd.). Ein zentrales Moment, welches in Erhebungssituationen zu Reaktanz führen kann, bildet die Anwesenheit von Forschenden in der Erhebungssituation und ihre Einflussnahme auf eben diese Situationen.

Die vermutete Reaktivität durch meine Datenerhebungsverfahren stellte für die ökonomischen LaborforscherInnen insofern ein Problem dar, da sie befürchteten, dass meine Methoden der Datenerhebung nachträglich Konsequenzen auf ihre eigene Datenerhebung im Experimentallabor ausüben könnten. Die verschiedenen Verfahren wurden dabei von Seiten der Laborforscher als unterschiedlich reaktiv betrachtet. Das Problem bestand somit nicht in der Auswirkung der Reaktanz meiner Methoden auf die Qualität meiner eigenen Daten. Meine Verfahren wurden stattdessen in unterschiedlichem Grade als potenzieller Störfaktor für die Erhebung meiner ForschungskollegInnen und anderer WissenschaftlerInnen angesehen, die den ProbandInnenpool des Experimentallabors nutzten. Es handelte sich somit um ein Problem der „Laborhygiene“, welches den Ansprüchen der Forschenden folgt, potenzielle Störfaktoren zu kontrollieren und auszuschließen. Für meine eigenen Erhebungen wurde diese Art von Reaktanz jedoch trotzdem zum Einflussfaktor, da sie bestimmte Arten der Datenerhebung meiner Forschung erschwerte.

In einem ersten Schritt erfolgt eine graduelle Einordnung der Erhebungsverfahren hinsichtlich der Stärke der Reaktivität, die die wirtschaftswissenschaftlichen ForscherInnen dem Einsatz der verschiedenen Datenerhebungsverfahren zuschrieben. Abbildung 4.3 dient der Illustration dieses Aspekts. In einem nächsten Schritt wird eine eigene Einordnung der genutzten Verfahren der Datenerhebung hinsichtlich ihrer reaktiven Wirkung auf die Ergebnisproduktion dargestellt und in Abbildung 4.4 veranschaulicht.

Die Nutzung von prozessproduzierten Daten und Dokumenten stellte keine Einflussnahme meinerseits dar und war aus diesem Grund unproblematisch. Auch die Kundgabe, dass die gemeinsamen Projektsitzungen zugleich zur Datenerhebung genutzt werden würden, war für die ökonomischen ForscherInnen nicht beanstandenswert. Die Interviews mit den ProbandInnen wurden nach Abschluss der Experimente geführt und gefährdeten die Datenerhebung im Labor aus Sicht der experimentellen WirtschaftsforscherInnen nicht. Auch Beobachtungen im Labor konnte ich alleine und ohne Probleme durchführen. Ich wurde jedoch dazu angehalten, mich ,unauffällig“ zu verhalten. Eine weitere beobachtende Person wurde anfangs nur unwillig akzeptiert, später jedoch toleriert und nicht weiter beanstandet.

Der zentrale Diskussionsgegenstand, der die Frage der Reaktivität von Methoden meiner Datenerhebung überhaupt erst deutlich als Problem offenbarte, 
war mein Wunsch, Videodaten im Labor zu erheben. Die Gefahr wurde von den wirtschaftswissenschaftlichen LaborforscherInnen darin gesehen, dass meine Datenerhebung eine Irritation im ProbandInnenpool hervorrufen könnte, welche in nachfolgenden Experimenten als Störgröße zu einer Verzerrung der Ergebnisse führen könnte. ${ }^{31}$ Die Frage, ob oder ob nicht Videokameras als Datenerhebungsmittel eingesetzt werden konnten, war somit eine Frage der „Laborhygiene“. Die Diskussion dauerte über sechs Monate an, endete jedoch abschließend erfolgreich, sodass eine erste Erhebungsreihe von drei Experimenten durchgeführt werden konnte. $^{32}$

Abb.4.3 Reaktivität der

Verfahren aus Sicht der

Laborforschenden

prozessproduzierte Dokumente

\author{
Interviews
}

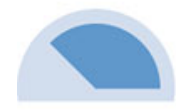

\title{
Beobachtung
}

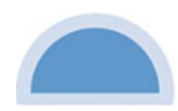

Videoaufnahmen

\section{Eigene Einschätzung der Reaktivität der Erhebungsverfahren}

Ausgehend von der obigen Definition der Reaktivität werden die prozessproduzierten Daten, die im Rahmen der Experimente ohne weiteres Zutun entstanden sind, als nicht-reaktiven Verfahren der Datenerhebung eingeordnet. Es handelt sich dabei um die quantitativen Daten der Experimente. Hinsichtlich der Analyse des Rahmenbildungsprozesses werden auch die standardisierten Fragebögen

\footnotetext{
${ }^{31}$ Es wurde von den Forschenden befürchtet, dass ProbandInnen annehmen könnten, dass sie nun heimlich gefilmt werden würden oder irritiert wären, da sie nun nicht mehr gefilmt würden, obwohl dies bei ihrem letzten Experiment jedoch der Fall gewesen war.

${ }^{32}$ Im Rahmen der Datenerhebung des qualitativen Experiments war es in der Folge deutlich leichter, eine erneute Videodatenerhebung durchzusetzen.
} 
als nicht reaktive Erhebungsverfahren eingeordnet. Hinzu kommen noch die verschiedenen schriftlichen Dokumente, die im Prozess des Experiments eingesetzt werden.

Eine geringe Reaktivität zeigt sich bei den Videoaufnahmen. Visuell wahrnehmbare Reaktanz zeigte sich vorrangig zu Beginn der Erhebungen: So lassen sich in Videodaten der Deckenkamera und Webcams zu Beginn des Sessions vereinzelt ProbandInnen entdecken, die zur Raumdecke hinaufschauen oder in die Kamera blicken. ${ }^{33}$ Auch in den Aufnahmen der Webcams im weiteren Verlauf der Aufzeichnung ist kein stark reaktives Verhalten zu erkennen. In den Interviews mit ProbandInnen im direkten Anschluss an diese Experimente wurde diese Frage des Einflusses der Videoaufnahme ebenfalls thematisiert. Die ProbandInnen gaben in der Selbstauskunft im Interview an, dass sie sich hinsichtlich ihrer Handlungen kaum durch die Videoaufzeichnung beeinflusst fühlten. Aus diesem Grund werden die Videoaufzeichnungen als wenig reaktives Verfahren betrachtet.

Die direkte Beobachtung von ProbandInnen im Labor erwies sich als unterschiedlich reaktives Verfahren und hing maßgeblich von der eingenommenen Beobachtungsposition ab. Anfangs beobachtete ich das allgemeine Laborgeschehen von einer recht fixen Position aus - meist aus der Position des Arbeitsplatzes der ExperimentatorInnen. Dies führte im Labor zu keiner merklichen Reaktanz bei der Erhebung von Beobachtungsdaten. Beobachtungen spezifischer ProbandInnen und ihres detaillierten Vorgehens waren aufgrund der hohen Trennwände jedoch nur möglich, wenn ich eine Beobachtungsposition direkt hinter dem Rücken der ProbandInnen einnahm. In dieser Position erwies sich die Beobachtung als stark reaktives Verfahren: Die ProbandInnen drehten sich vermehrt zu mir um oder verharrten in einer Position. Es war somit nicht möglich, längere Beobachtungen aus dieser Position heraus zu tätigen.

In der Rolle der Experimentatorin führte ich, wie bereits beschrieben, eine ganze Reihe von Experimenten durch. Bestandteil dieser Aufgabe war auch der regelmäßige Rundgang durch das Labor oder die Interaktion mit einzelnen ProbandInnen. Dadurch war es mir möglich, vielfältige Beobachtungen von ProbandInnen und ihrem spezifischen Verhalten zu tätigen, ohne dass dadurch die

\footnotetext{
${ }^{33}$ Aus datenschutzrechtlichen Gründen wurden alle ProbandInnen bereits im Zuge der Einladung zu den Experimenten darüber informiert, dass diese mit Video aufgezeichnet werden würden. Darüber hinaus wurden an der Tür des Labors sowie auch im Labor selbst Hinweisschilder mit der Aufschrift „Achtung! Videoüberwachung“ angebracht. Vor ihrem Eintritt ins Labor unterzeichnete zudem jeder der Teilnehmenden eine Einverständniserklärung. Diese sowie der Textabschnitt in der Einladung wurden in Rücksprache mit den Datenschutzbeauftragten der Universität verfasst und durch diese genehmigt.
} 
natürliche Situation des Laborgeschehens stark beeinflusst wurde. Die Beobachtungsdaten, die in Zusammenarbeit mit den wirtschaftswissenschaftlichen ForscherInnen generiert wurden, weisen ebenfalls eine unterschiedlich starke Reaktivität auf. Die Fälle, in denen ich durch meine Projektarbeit selbst aktiv in Diskussionszusammenhänge eingebunden war, sind deutlich stärker von mir beeinflusst worden, als solche, in denen ich primär als teilnehmende Beobachterin agierte.

Ich selbst betrachte Interviews als durchaus reaktive Verfahren der Datenerhebung. Einerseits, da Forschende hier als Kommunikationspartner an der interaktiven Hervorbringung der Situation direkt beteiligt sind. Darüber hinaus obliegt es den Forschenden im Zuge dieser Gespräche, bestimmte Themen zu fokussieren und andere auszusparen, die womöglich gegenteilige Ergebnisse liefern würden. Um die Natürlichkeit und Eigengesetzlichkeit eines Untersuchungsphänomens $\mathrm{zu}$ untersuchen und Interaktionenprozesse sowie situative Vollzüge zu betrachten, sind Interviews aus diesem Grund nur begrenzt geeignet. Sie liefern keine natürlichen Daten über das Interaktionsgeschehen, sondern Beschreibungen durch Handelnde. ${ }^{34}$

\subsection{Wissenssoziologische Gattungsanalyse als gegenstandsadäquates Verfahren}

Wie die bisherige Darstellung aufzeigt, wurden vielfältige Daten erhoben. Alle folgten dem Ziel, die soziale Veranstaltung des wirtschaftswissenschaftlichen Laborexperiments in seiner natürlichen Eigengesetzlichkeit und seinen unterschiedlichen Dimensionen zu erfassen. Während die Ergebnisse der verschiedenen Datenerhebungsmethoden einerseits alle mein ethnographisches Wissen über die Situation bereicherten, war es analytisch lange Zeit problematisch, ein Konzept auszumachen, welches fähig war, diese Diversität der Datensorten aufzufangen und zugleich den spezifischen Erklärungsleistungen der unterschiedlichen Daten gerecht zu werden. Der Anspruch der Gegenstandsadäquanz als Gütekriterium für

\footnotetext{
${ }^{34}$ Trotz dieser Einschränkungen wurden Interviews gewinnbringend im Forschungsprozess eingesetzt, um die Entscheidungshintergründe der Handelnden, ihre Erwartungen und Vorerfahrungen mit Experimenten besser zu verstehen. Die Struktur der Interviews orientierte sich dabei an einem Leitfaden. Dieser diente jedoch vorrangig dazu, Themenbereiche für die Interviews und eine grobe Ablaufstruktur festzulegen. Der Leitfaden wurde dabei sukzessiv im Zuge meines Erkenntnisfortschritts verändert. Zur Vermeidung von reaktiven Intervieweffekten wurde die Interviewsituation mehrfach verändert und geprüft, ob dies das Antwortverhalten der Interviewten beeinflussen würde.
} 
Abb.4.4 Graduelle

Einordnung des Grades der

Reaktivität der eingesetzten

Prozessproduzierte Daten

Methoden

Beobachtung der

LaborforscherInnen (1)

\section{Beobachtung der \\ ProbandInnen (1)}

Videoaufnahmen

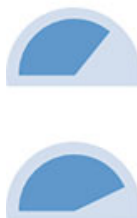

Interview

Beobachtung der

ProbandInnen (2)

\section{Beobachtung der}

LaborforscherInnen (2)

die Forschung war dabei jedoch nicht nur für die Suche geeigneter Verfahren der Datenerhebung leitend, sondern auch für die Auswahl des passenden Analyseverfahrens. Der folgende Abschnitt zeigt auf, dass die Auswahl der Gattungsanalyse als wissenssoziologische Analysemethode aus eben diesem Anspruch herrürte. ${ }^{35}$

Die Auseinandersetzung mit dem Thema des Projekts, in das ich eingebunden war, bedingte in einem ersten Schritt eine Fokussierung auf die Begriffe des Rahmens und der Rahmung sowie zugleich eine Auseinandersetzung mit den soziologischen und ökonomischen Konzepten der Rahmung. Aus einer qualitativen soziologischen Perspektive war der Goffmansche Begriff der Rahmung und

\footnotetext{
${ }^{35}$ Der höhere Abstraktionsgrad der Gattungsanalyse auf der Ebene der Binnen- und Außenstruktur wurde zudem maßgeblich durch die Reflexion der Kombinationsmöglichkeiten von Grounded Theory und Ethnomethodologie angestoßen (siehe dazu Abschnitt 3.3.2). Zugleich muss darauf verweisen werden, dass der Einfluss der Grounded Theory und ihrer forschungsleitenden Annahmen für die Suche und das Finden der passenden Analysemethode auch in diesem Abschnitt im Sinne einer reflexiven Perspektive auf den eigenen Forschungsprozess (Breuer 2009) hätte diskutiert werden können.
} 
seine Fokussierung auf das Interaktionsgeschehen der zentrale Ausgangspunkt meiner konzeptionellen Suchbewegung.

Meine eigene Forschungsperspektive war dabei zu Beginn des Projekts maßgeblich durch die qualitative Wissenssoziologie, die Ethnomethodologie und den Sozialkonstruktivismus von Berger und Luckmann geprägt. Hinsichtlich der Auseinandersetzung mit dem Untersuchungsphänomen und dem hohen Detailanspruch meiner Analysen stellte die Forschungsperspektive der Ethnomethodologie sowohl konzeptionell als auch hinsichtlich der analytischen Mentalität einen zentralen Orientierungspunkt dar, der sich durch meine bisherigen Forschungsarbeiten ergab. Die ethnomethodologische Perspektive und das begriffliche Instrumentarium, welches mit dieser verbunden ist, eröffnete die Möglichkeit, die situative Hervorbringung des Phänomens detailliert zu erfassen und darzustellen. Die Analyse empirischer Situationen zeigte zugleich wiederholt auf, wie die Handelnden aktiv auf bestimmte Wissensbestände Bezug nahmen. Diese bildeten zentrale Kontextelemente und Hintergrundannahmen. Gerade die Daten der Interviews und die Diskussionen der Projektgruppe hinsichtlich der Konzeption und Planung der anstehenden Experimente verwiesen auf die Relevanz dieser Aspekte. Um die Spezifik des Laborexperiments abzubilden, erschien es jedoch entscheidend, herauszuarbeiten, welchen Einfluss Wissenshintergründe und typisierte Annahmen im Vollzug des Laborexperiments selbst hatten. In meinen früheren Artikeln und Vorträgen, wurden einige diese Aspekte als rahmende Faktoren für eine ethnomethodologisch orientierte Untersuchung dargestellt. Eine gezielte Integration in die Analyse und eine Systematisierung dieser Gesichtspunkte war dabei jedoch nicht möglich.

Die soziologische Gattungsanalyse ${ }^{36}$ stellte nun eine Lösung für dieses Problem dar. Wie im Abschnitt 3.3 beschrieben, ist sie eine wissenssoziologische

\footnotetext{
${ }^{36}$ Wie im Theorieteil dieser Arbeit dargestellt ist, bildet der Sozialkonstruktivismus von Berger und Luckmann (1980 [1969]) eine zentrale Grundlage der Luckmannschen Gattungsanalyse (Abschnitt 3.3). Es wird davon ausgegangen, dass Menschen die Wirklichkeit, die ihnen objektiv erscheint, in ihren Handlungen selbst herstellen. Einen weiteren Orientierungspunkt in der Entwicklungsgeschichte der Gattungsanalyse stellt die Konversationsanalyse dar. Diese fußt, hinsichtlich ihrer methodologischen Prämissen wiederum maßgeblich auf der Ethnomethodologie (Abschnitt 3.2). Ethnomethodologie, Konversationsanalyse und Gattungsanalyse eint, dass sie üblicherweise alltägliche Handlungen untersuchen. Dabei arbeiten sie die Muster und Methoden heraus, mittels welcher diese Phänomene in ihrer Hervorbringung die spezifische soziale Ordnung produzieren und reproduzieren und damit zugleich die gesellschaftliche Wirklichkeit der Kultur, der sie entstammen. Sie beziehen sich damit auf Wissensinhalte, die die Handelnden im Rahmen ihrer Sozialisation erworben haben und auf die sie in der praktischen Bewältigung ihrer alltäglichen Angelegenheiten zurückgreifen.
} 
Analysemethode, die für die Untersuchung von musterhaften Formen von Kommunikationsphänomenen konzipiert wurde. Diese musterhaften Formen werden als kommunikative Gattungen bezeichnet. Die soziologische Gattungsanalyse kann dem Bereich der Wissenssoziologie zugeordnet werden, da sie sich dem Wissen der Handelnden und seiner situationsangemessenen Nutzung widmet. Die Gattungsanalyse besitzt eine starke konzeptionelle Verortung im Feld des Sozialkonstruktivismus von Berger und Luckmann und der sprachsoziologischen Orientierung von Luckmann und ist von einem sequenzanalytischen Vorgehen geprägt, welches auch in ethnomethodologischen und konversationsanalytischen Studien vorliegt. Der entscheidende Zugewinn durch die wissenssoziologische Gattungsanalyse besteht jedoch in der Einbeziehung des Kontexts und der Kontextbedingungen auf der Analyseebene der Außenstruktur.

Wie die Ausführungen zur Datenerhebung zeigten, bestand ein grundlegendes Problem darin, im Labor fruchtbare Daten zu erheben, obwohl nur wenig verbalsprachliche Kommunikation festgehalten werden konnte. Es scheint aus diesem Grund merkwürdig, dass eine Analysemethode als besonders passend erscheinen soll, die sich gerade Dingen widmet, die im Untersuchungsbereich nur begrenzt erhoben werden konnten und der Spezifik des Beobachtunggegenstandes nicht gerecht zu werden scheinen. Eine klassische Gattungsanalyse nach Luckmann (1986) hätte im wirtschaftswissenschaftlichen Experimentallabor aus eben diesen Gründen nicht analytisch gewinnbringend durchgeführt werden können.

Führt man sich jedoch in Erinnerung, was das Konzept der kommunikativen Gattung genauer bezeichnet, dann scheint die Fixierung auf verbalsprachliche Kommunikationsaspekte keine grundlegende Bedingung. Gattungen stellen als „Institutionen der Kommunikation“ (Knoblauch \& Luckmann 2000: 539) Lösungen für kommunikative Phänomene bereit. Sie sind dabei Teil des gesellschaftlichen Wissensvorrats und bilden vorhersagbare Muster aus, an denen sich die Handelnden in typischer Weise orientieren. Sie bilden dabei Wissensbezüge ab, die sich als wiederkehrende und charakteristische Momente dieser Kommunikationsphänomene zeigen und aus diesem Grund zugleich einer wissenschaftlichen Analyse zugänglich werden.

Das, was die Gattungsanalyse in ihren unterschiedlichen Ebenen eigentlich thematisiert, sind Aspekte dieser Wissensbezüge und Wege ihrer typischen Realisierung und Aktivierung in den empirischen Situationen. Die Außenstruktur thematisiert dabei die institutionellen Zusammenhänge, in welche das kommunikative Phänomen eingebettet ist. Auf der Ebene der situativen Realisierung werden die Merkmale des situativen Kontexts verortet und solche, die der Koordination der kommunikativen Handlung dienen. Die Binnenstruktur hingegen bezieht sich auf typische Merkmale des Kommunikationsphänomens, die nicht 
durch die interaktive Handlungsabfolge geleitet sind (Günthner \& Knoblauch 2007: 57 f.).

Auch die Mitglieder einer Wissenschaftskultur erwerben im Verlauf ihrer Sozialisation das notwendige Wissen über zentrale kommunikative Formen. Im Sinne des weiten Kommunikationsbegriffs des kommunikativen Konstruktivismus (Knoblauch 2013, 2017) handelt es sich dabei um Wissen über typische Muster kommunikativer Handlungen, die zur Produktion und Interpretation herangezogen werden oder zur typischen Erzeugung kommunikativer Inhalte dienen.

Die von Schnettler und Knoblauch (2007) veröffentlichte Untersuchung zu PowerPoint Präsentationen zeigte eindrücklich, auf welche fruchtbare Weise die Gattungsanalyse auf Phänomene angewandt werden kann, bei denen die Fokussierung auf die verbalsprachlichen Aspekte dem analysierten Kommunikationsphänomen nicht gerecht wird. ${ }^{37}$ Es wird davon ausgegangen, dass die Bedeutung eines Kultur- oder Kommunikationsphänomens ${ }^{38}$ erst im Kontext seiner performativen Realisierung entsteht. ${ }^{39}$ Es gilt somit in der empirischen Analyse die prozessual entstehende Ordnung aufzuzeigen, da sich erst in der Realisierung und somit in der Performanz die eigentliche Ordnung entfaltet (Schnettler et al. 2007: 20). Die Autoren verweisen selbst darauf, dass die Videographie (und die Videoperformanzanalyse) diesen Anspruch aufgreifen. Videoaufnahmen ablaufender Kommunikationsprozesse ermöglichen es, die vielfältigen Elemente und Aspekte der Situationen einzufangen und für eine Analyse zu konservieren.

Eine theoretische und analytische Erklärung dafür, wie Performanz die Ebene des Situativen überschreiten und damit zugleich auf Institutionalisierungen verweisen kann, bietet der kommunikative Konstruktivismus (Knoblauch 2013, 2017). Den Kernbegriff des kommunikativen Konstruktivismus stellt das kommunikative Handeln dar. Nach Knoblauch ist kommunikatives Handeln als Wirkhandeln zu verstehen und bezieht dabei den Körper mit ein. ${ }^{40}$ Die Erzeugung von Objektivierungen ist an den Körper gebunden - durch ihn wird der soziale

\footnotetext{
${ }^{37}$ Auf diese Studie wurde ausführlicher im Abschnitt 3.4.4 eingegangen.

${ }^{38}$ Das ist nicht als Unterscheidung, sondern als Spezifizierung gedacht, da Kommunikationsphänomene ebenso Kulturphänomene sind.

${ }^{39}$ Es zeigt sich hier eine deutliche Nähe zu dem „Konzept des Doing“ der Ethnomethodologie.

${ }^{40}$ Die Trennung von instrumentellem und kommunikativem Handeln, wie sie Habermas (1981) vorschlägt, wird im kommunikativen Konstruktivismus aufgehoben. Kommunikatives Handeln ist damit immer zugleich auch instrumentelles Handeln. Zu weiteren Ausführungen über den kommunikativen Konstruktivismus sei auf die Ausführungen im Theorieteil im Abschnitt 3.4 verwiesen.
} 
Sinn des kommunikativen Handelns sichtbar. Das Wirken und die so entstehenden Objektivierungen (er)klären damit zugleich, wie Materialität und Technik im Rahmen des kommunikativen Handelns thematisiert werden können.

Legt man diesen weiten Kommunikationsbegriff und das Verständnis von kommunikativem Handeln als Wirkhandeln der Analyse kommunikativer Gattungen zugrunde, eröffnen sich neuartige Anwendungsmöglichkeiten. Empirische Untersuchungen des kommunikativen Handelns und seiner strukturellen Muster betrachten somit Handlungsverläufe und Objektivierungen in ihrem spezifischen zeitlichen Vollzug. Objektivationen lösen dabei den Sinn vom körperlichen Vollzug seiner Erzeugung ab und verfestigen kommunikative Inhalte und damit das Wissen, welches mit ihnen einhergeht. Durch die Objektivationen können nun auch materielle Aspekte als Teile der typischen strukturellen Muster der kommunikativen Gattungen erfasst werden. Für die Anwendung der Gattungsanalyse können sie nun einen anderen Stellenwert in der Orchestrierung sozialer Situationen einnehmen. Zu Unrecht wurde ihnen bisher stets nur der Platz sozialer Kontextelemente auf der Ebene der situativen Realisierung zugewiesen.

Die Betrachtung des ökonomischen Laborexperiments aus der Perspektive der kommunikativen Gattungsanalyse zielt - basierend auf der dargestellten konzeptionellen Einbettung - darauf ab, das Besondere dieser Kommunikationssituation herauszuarbeiten und zugleich ihre typischen ordnungsstiftenden Muster zu entdecken. Ähnlich wie in der Analyse von PowerPoint Präsentationen als kommunikative Gattung muss dabei der Performanz und der Orchestrierung einzelner Handlungselemente besondere Aufmerksamkeit geschenkt werden. Die beobachtbaren Interaktionssituationen im Labor bildeten den Dreh- und Angelpunkt der Analyse. Ganz im Sinne der Grounded Theory Methodologie kann der Forschungsprozess als iterativ-zyklisch bezeichnet werden und wechselte mehrfach zwischen Datenerhebung, -auswertung und der schrittweisen Weiterentwicklung der eigenen Konzepte.

Wie im Methodenteil ausführlich dargestellt, wurden im Zuge des Forschungsprozesses eine Reihe unterschiedlicher Datensorten erhoben. Grund war eine möglichst gegenstandsadäquate Auseinandersetzung mit dem Forschungsgegenstand. Einerseits sollten dabei die Eigenheit des Phänomens und der Feldakteure geachtet und zugleich differenzierte Daten generiert werden, um die Forschungsfrage nach der Produktion und Aufrechterhaltung der charakteristischen Rahmung des ökonomischen Laborexperiments bestmöglich zu analysieren und zu beantworten. Es wird dabei die analytische Trennung der Gattungsanalyse in drei Betrachtungsebenen aufgenommen und für die Strukturierung der Analyse genutzt. Die unterschiedlichen Datensorten gehen dabei in die verschiedenen Ebenen der Analyse in unterschiedlichem Maße ein. Einen Überblick über die Nutzung der Daten gibt die nachfolgende Tabelle 4.1: 


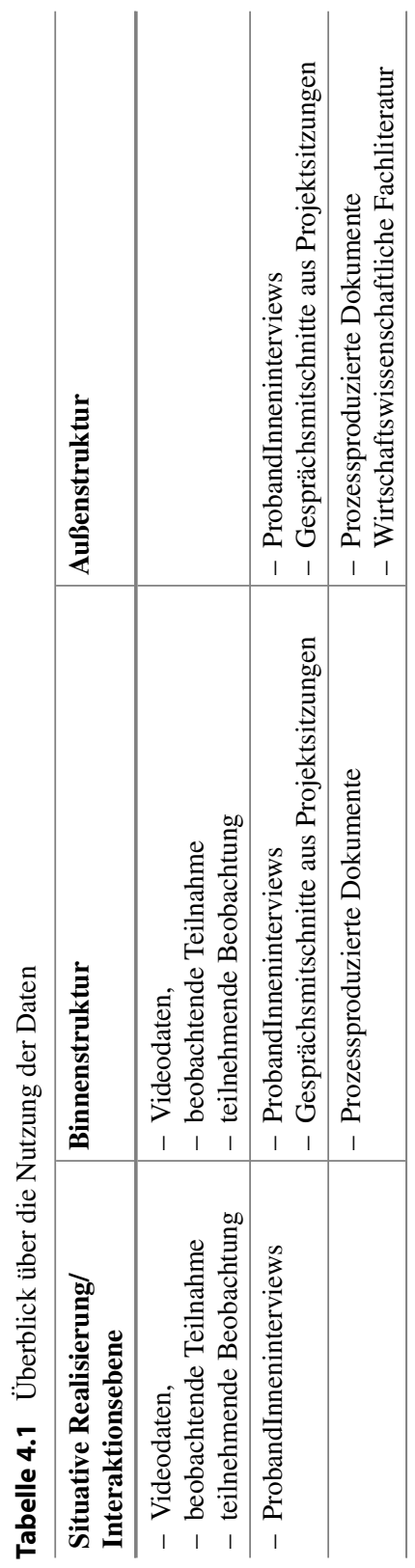


In der Auseinandersetzung mit dem empirischen Material gilt es nun zugleich, die Adäquanz der Gattungsanalyse für die eigene Forschung zu überprüfen. Gestützt auf den erweiterten Kommunikationsbegriff des kommunikativen Konstruktivismus, erscheint beispielsweise die klassische gattungsanalytische Zuordnung vielfältiger nichtsprachlicher und materieller Aspekte auf der Ebene der situativen Realisierung als problematisch. Denn der Position des kommunikativen Konstruktivismus folgend, können Objektivationen nicht nur durch sprachliche Entäußerungen gebildet werden. Auch materielle Aspekte der Handlungssituation können als Objektivationen kommunikativer Handlungen wirken und dies in so systematischer Weise, dass andere Zuordnungen zu den drei Ebenen der Gattungsanalyse als analytisch fruchtbar erscheinen. Einige Anstöße für Veränderungen und Erweiterungen der Gattungsanalyse, die sich aufbauend auf der Auseinandersetzung mit dem empirischen Material ergeben, werden im Anschluss an die empirische Analyse diskutiert. Im folgenden empirischen Teil der Arbeit wird nun aufgezeigt, wie der Ansatz der Gattungsanalyse für meine eigene Untersuchung nutzbar gemacht wurde und welcher entscheidende Mehrwert sich dadurch ergab.

Open Access Dieses Kapitel wird unter der Creative Commons Namensnennung 4.0 International Lizenz (http://creativecommons.org/licenses/by/4.0/deed.de) veröffentlicht, welche die Nutzung, Vervielfältigung, Bearbeitung, Verbreitung und Wiedergabe in jeglichem Medium und Format erlaubt, sofern Sie den/die ursprünglichen Autor(en) und die Quelle ordnungsgemäß nennen, einen Link zur Creative Commons Lizenz beifügen und angeben, ob Änderungen vorgenommen wurden.

Die in diesem Kapitel enthaltenen Bilder und sonstiges Drittmaterial unterliegen ebenfalls der genannten Creative Commons Lizenz, sofern sich aus der Abbildungslegende nichts anderes ergibt. Sofern das betreffende Material nicht unter der genannten Creative Commons Lizenz steht und die betreffende Handlung nicht nach gesetzlichen Vorschriften erlaubt ist, ist für die oben aufgeführten Weiterverwendungen des Materials die Einwilligung des jeweiligen Rechteinhabers einzuholen.

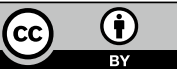

